\title{
EXISTENCE OF GLOBAL ATTRACTOR FOR A NONAUTONOMOUS STATE-DEPENDENT DELAY DIFFERENTIAL EQUATION OF NEURONAL TYPE
}

\author{
CINZIA ELIA, ISMAEL MAROTO, CARMEN NÚÑEZ, AND RAFAEL OBAYA
}

\begin{abstract}
The analysis of the long-term behavior of the mathematical model of a neural network constitutes a suitable framework to develop new tools for the dynamical description of nonautonomous state-dependent delay equations (SDDEs). The concept of global attractor is given, and some results which establish properties ensuring its existence and providing a description of its shape, are proved. Conditions for the exponential stability of the global attractor are also studied. Some properties of comparison of solutions constitute a key in the proof of the main results, introducing methods of monotonicity in the dynamical analysis of nonautonomous SDDEs. Numerical simulations of some illustrative models show the applicability of the theory.
\end{abstract}

\section{INTRODUCTION}

The analysis of nonautonomous differential equations constitutes a complex field in Mathematics on which many researchers, starting from Poincaré, have actively worked. A large quantity of problems are relevant not only because of their theoretical interest, but also due to their fundamental role in the more accurate mathematical modeling of many different actual phenomena. Generally speaking, the goal is to understand the way in which the intrinsic dynamics of the dynamical system determined by a nonautonomous equation, which is due to the explicit time dependence of the law, affects the behavior of the phenomenon under analysis. Quite often the dynamical scenario described by the analysis reproduces well-known patterns of the autonomous case; but sometimes there appear new scenarios which cannot occur in the autonomous or periodic cases, with a high degree of complexity.

Certain properties of regularity on the time variation of the functions determining the equation allow us to include it in a collective family of equations of the same type, whose solutions define a continuous or random flow or semiflow of skewproduct type. This procedure has been the initial point to develop a wide collection of dynamical techniques, both analytical and numerical, which constitute the core of a robust theory. Suitable references can be those of Sell [31], Sacker and Sell [29], Chow and Leiva [7, 8], Arnold [1], Shen and Yi [32], Cheban et al [6], Kloeden and Rassmussen [21], Carvalho et al [5], Caraballo and Han [3], Johnson et al [18], as well as the works cited therein.

2010 Mathematics Subject Classification. 37B55, 34K20, 37B25, 34K14, 92B20 .

Key words and phrases. Nonautonomous state-dependent delay differential equation, global attractor, neural network.

Partly supported by Ministerio de Economía y Competitividad / FEDER under project MTM2015-66330-P, by Ministerio de Ciencia, Innovación y Universidades under project RTI2018096523-B-I00, and by European Commission under project H2020-MSCA-ITN-2014. 
The main objective of this paper is to provide new tools for the dynamical description of nonautonomous functional differential equations with state-dependent delay (SDDEs for short), focusing in models of neural networks. We will show that some methods which have been frequently used in the analysis of the long term dynamics of neural networks with time-dependent delay can be adapted to the case of state-dependent delay. The weak regularity of the solutions of the SDDEs with respect to the initial states makes this extension not trivial.

Our tools are, roughly speaking, two. The first one is to define and describe global and pullback attractors in this setting, as well as to establish criteria ensuring their existence and providing a global description of their shapes. As far as we know, this is the first time that a theory on the existence and dynamical properties of attractor sets is given in the setting of nonautonomous SDDEs. As second tool, we introduce arguments of the theory of nonautonomous monotone dynamical systems in the study of nonautonomous SDDEs. We compare our SDDEs with simpler types of nonautonomous ordinary differentail equations which satisfy a quasimonotone condition to determine the area containing the global attractor. In a similar way, we compare the linearized families of SDDEs equations with families of functional equation for which the delay is just time-dependent, in order to obtain appropriate bounds for the upper Lyapuov exponents of minimal sets. In same cases these bounds show that the attractor has a simple shape and the existence of globally exponentially stable recurrent solutions. To our knowledge this is the first time that methods of nonautonomous monotone dynamical systems are applied in the context of SDDEs.

A large number of researchers have been interested in the dynamics induced by SDDEs, motivated both by its high theoretical interest and by the increasing number of models of applied sciences which respond to this pattern. Among them, we can mention Hartung [12, 13], Wu [38], Hartung et al. [14], Mallet-Paret and Nussbaum [23], $\mathrm{Hu}$ and $\mathrm{Wu}[20], \mathrm{Hu}$ et al. [19], Walther [35, 36], He and de la Llave [15, 16], Krisztin and Rezounenko [22] and Maroto et al. [24, 25], as well as the many references therein. In particular, the regularity properties of the solutions of families of SDDEs which we will use in this paper are described in [24, 25].

Next we describe briefly the contents of this paper, which are organized in three sections.

We begin Section 2 by recalling some standard notions of topological dynamics, as well as the concepts of global attractor, exponentially stable set and upper Lyapunov exponent. The last three definitions are referred to a skew-product semiflow $\left(\Omega \times X, \zeta, \mathbb{R}^{+}\right)$projecting on a flow $(\Omega, \sigma, \mathbb{R})$, where $\Omega$ is a compact metric space and $X$ is a Banach space. For the main purposes of this paper, $\Omega$ will be the hull of the almost periodic coefficients of the SDDEs which models our neural network and $\sigma$ will be the time-translation flow on $\Omega$ (see Section 3.1 for the details). This hull procedure will allow us to construct a family of SDDEs of the form

$$
\dot{y}(t)=F\left(\omega \cdot t, y(t), y\left(t-\tau\left(\omega \cdot t, y_{t}\right)\right)\right), \quad t \geq 0
$$

for $\omega \in \Omega$, where $\{\omega \cdot t \mid t \in \mathbb{R}\}$ is the $\sigma$-orbit of the point $\omega \in \Omega$. (The standard regularity conditions assumed on the map $F$ and the delay $\tau$ are described in detail in Section 2). The space $X$ will be $W_{2}^{1, \infty}:=W^{1, \infty}\left([-r, 0], \mathbb{R}^{2}\right)$, where $r>0$ is the maximum delay. And the (local) semiflow $\zeta$ will be given by

$$
\zeta: \mathcal{U}_{2} \subseteq \mathbb{R}^{+} \times \Omega \times W_{2}^{1, \infty} \rightarrow \Omega \times W_{2}^{1, \infty}, \quad(t, \omega, x) \mapsto(\omega \cdot t, u(t, \omega, x)),
$$


with $u(t, \omega, x)(s):=y(t+s, \omega, x)$ for $s \in[-r, 0]$, where $y(t, \omega, x)$ is the solution of the equation corresponding to $\omega \in \Omega$ and $x \in W_{2}^{1, \infty}$ with $y(s, \omega, x)=x(s)$ for $s \in[-r, 0]$. The map $\zeta$ may be noncontinuous on $[0, r] \times \Omega \times W_{2}^{1, \infty}$. However, it satisfies enough continuity properties to be still a valuable tool in the long-term analysis to be carried out. As a matter of fact, it has a continuous restriction to the compatibility set $\mathcal{C}_{n}^{0}:=\left\{(\omega, x) \in \Omega \times C_{n}^{1} \mid \dot{x}\left(0^{-}\right)=F(\omega, x(0), x(-\tau(\omega, x)))\right\}$. This property and some others are summarized in Section 2. The set $\mathcal{C}_{n}^{0}$ is closed and invariant, but in general not a differentiable manifold in this nonautonomous setting. One more result, concerning the monotonicity properties of $\zeta$ as well as comparison results for "ordered" families of SDDEs satisfying a quasi-monotonicity condition, completes the section. It adapts an already classical result of Smith [33].

Section 3 contains the core results of this paper. It begins with the description of the model for the biological network, given by a two-dimensional system of nonautonomous SDDEs with almost periodic coefficients. It is a simplified model for two groups of neurons: the internal action of each group is assumed to be instantaneous, while the action onto the other group is assumed to be delayed, and with state-dependent delay. As said above, the hull construction includes this system in a family of the type (1.1). Therefore, we can define a semiflow, part of whose orbits are defined exactly by the solutions of the initial system. The topological dynamics techniques allow us to show the existence of a global attractor $\mathcal{A}$, which is determinant to understand the long term dynamics. It is important to emphasize that the classical theory of attractors is carried out in the autonomous case, while here we are dealing with a nonautonomous (and statedependent) problem: the fundamental tool to make this extension possible is the skew-product formalism. It is also remarkable that the problems that the absence of global continuity causes, both in the definitions and in the proofs of the results, can be solved thanks to the actual continuity properties.

Section 3 also contains a brief explanation of the way in which the existence and properties of the global attractor ensure the existence and some properties of the so-called pullback attractor (see e.g. [5]) for the process defined from the initial nonautonomous SDDE. In addition, under the additional hypotheses of the exponential stability of the minimal sets of $\Omega \times W_{2}^{1, \infty}$, the attractor $\mathcal{A}$ turns out to agree with the graph of a continuous function $a: \Omega \rightarrow W_{2}^{1, \infty}$, which we call copy of the base. Moreover, all the semiorbits are exponentially attracted to $\mathcal{A}$. It is proved in $[25]$ that the exponential stability of a minimal set is equivalent to the negativeness of its upper Lyapunov exponent. A procedure which makes it easier to determine if this condition holds completes Section 3.

In Section 4 we carry out some numerical experiments for a particular model. First, using the comparison methods previously described, we delimit a region containing the global attractor. We perform simulations of the model under study both in the forward sense and in the pullback sense. The computer simulations suggest the existence of an attractor for the numerical method and show that the bounds we obtained for the containing area are quite accurate. We also check that the conditions ensuring that the global attractor is a copy of the base are fulfilled if we are more exigent in the choice of the delay, and give numerical evidence that the attractor we see in the simulations is indeed a copy of the base.

We finally point out that the ideas here developed shall be useful in the analysis of many other phenomena which can be modeled by nonautonomous SDDEs. 


\section{BASIC NOTIONS AND PROPERTIES}

The basic notions and some classical results on topological dynamics required in the paper are recalled in this section, whose contents may be found in Sell [31], Sacker and Sell [29, 30], Hale [11], Chow and Leiva [7, 8], Shen and Yi [32], and references therein.

Let $\Omega$ be a complete metric space with distance $d_{\Omega}$. A flow $(\Omega, \sigma, \mathbb{R})$ is defined by a Borel measurable map $\sigma: \mathbb{R} \times \Omega \rightarrow \Omega,(t, \omega) \mapsto \sigma(t, \omega)$ satisfying

$$
\text { (f1) } \sigma_{0}=\mathrm{Id}, \quad \text { (f2) } \sigma_{t+l}=\sigma_{t} \circ \sigma_{l} \text { for all } s, t \in \mathbb{R}
$$

where $\sigma_{t}(\omega):=\sigma(t, \omega)$ for all $t \in \mathbb{R}$ and $\omega \in \Omega$. The flow is continuous if $\sigma$ is continuous. The sets $\left\{\sigma_{t}(\omega) \mid t \in \mathbb{R}\right\},\left\{\sigma_{t}(\omega) \mid t \geq 0\right\}$ and $\left\{\sigma_{t}(\omega) \mid t \leq 0\right\}$ are respectively the orbit, positive or forward semiorbit and negative or backward semiorbit of the point $\omega \in \Omega$. If the forward (or backward) semiorbit is relatively compact, the omega-limit set (resp. alpha-limit set) of the point $\omega \in \Omega$ (or of its semiorbit) is the set of limits of sequences of the form $\left(\sigma_{t_{n}}(\omega)\right)$ with $\left(t_{n}\right) \uparrow \infty$ (resp. $\left.\left(t_{n}\right) \downarrow \infty\right)$. A Borel set $\mathcal{M} \subseteq \Omega$ is $\sigma$-invariant (or just invariant, if no confusion arises) if $\sigma_{t}(\mathcal{M})=\mathcal{M}$ for all $t \in \mathbb{R}$, and it is $\sigma$-minimal (or minimal) if it is compact, $\sigma$ invariant, and it contains properly no nonempty compact $\sigma$-invariant subset. Zorn's lemma ensures that every $\sigma$-invariant compact set contains a minimal subset; and clearly a compact $\sigma$-invariant subset is minimal if and only if each one of its semiorbits is dense in it. A flow $(\Omega, \sigma, \mathbb{R})$ is recurrent or minimal if $\Omega$ itself is minimal. A continuous flow $(\Omega, \sigma, \mathbb{R})$ is almost periodic if for any $\varepsilon>0$ there is a $\delta=\delta(\varepsilon)>0$ such that, if $\omega_{1}, \omega_{2} \in \Omega$ satisfy $d_{\Omega}\left(\omega_{1}, \omega_{2}\right)<\delta$, then $d_{\Omega}\left(\sigma_{t}\left(\omega_{1}\right), \sigma_{t}\left(\omega_{2}\right)\right)<\varepsilon$ for all $t \in \mathbb{R}$. The flow is local if the map $\sigma$ is defined and satisfies (f1) and (f2) on an open subset $\mathcal{U} \subseteq \mathbb{R} \times \Omega$ containing $\{0\} \times \Omega$.

As usual, we represent $\mathbb{R}^{ \pm}:=\{t \in \mathbb{R} \mid \pm t \geq 0\}$. A semiflow $\left(\Omega, \sigma, \mathbb{R}^{+}\right)$is given by a Borel measurable map $\sigma: \mathbb{R}^{+} \times \Omega \rightarrow \Omega,(t, \omega) \mapsto \sigma(t, \omega)$ satisfying (f1) and (f2) for all $t, s \in \mathbb{R}^{+}$; and it is continuous if $\sigma$ is continuous. Positive semiorbits and omega-limit sets are defined as above. A Borel subset $\mathcal{M} \subseteq \Omega$ is positively $\sigma$-invariant if $\sigma_{t}(\mathcal{M}) \subseteq \mathcal{M}$ for all $t \geq 0$. A positively $\sigma$-invariant compact set $\mathcal{M}$ is $\sigma$-minimal (or minimal) if it does not contain properly any positively $\sigma$-invariant compact set. If $\Omega$ is minimal, we say that the semiflow $\left(\Omega, \sigma, \mathbb{R}^{+}\right)$is minimal. The semiflow is local if the map $\sigma$ is defined, continuous, and satisfies (f1) and (f2) on an open subset $\mathcal{U} \subseteq \mathbb{R}^{+} \times \Omega$ containing $\{0\} \times \Omega$. In this case, the definitions of positively invariant set and minimal set are the same as above. In particular, they are composed of globally defined positive semiorbits, so that the restriction of the semiflow to one of these sets is global.

Let the semiflow $\left(\Omega, \sigma, \mathbb{R}^{+}\right)$be continuous. A point $\omega \in \Omega$ has a complete orbit in $\Omega$ if there exists a continuous map $\theta_{\omega}: \mathbb{R} \rightarrow \Omega$ such that $\theta_{\omega}(0)=\omega$ and $\sigma\left(t, \theta_{\omega}(s)\right)=$ $\theta_{\omega}(t+s)$ whenever $s \in \mathbb{R}$ and $t \geq 0$. If the corresponding negative semiorbit $\left\{\theta_{\omega}(t) \mid t \leq 0\right\}$ is relatively compact, then it has an alpha-limit set, defined as above. A set $\mathcal{M} \subseteq \Omega$ is $\sigma$-invariant if $\sigma_{t}(\mathcal{M})=\mathcal{M}$ for all $t \geq 0$. Note that this condition is quite stronger than the positively $\sigma$-invariance. It is not hard to prove that $\mathcal{M}$ is $\sigma$-invariant if and only if it is composed by complete orbits of its elements: see, e.g., Lemma 1.4 of Carvalho et al. [5]. In addition, a minimal set is $\sigma$-invariant, as easily deduced from the minimality itself. The same happens with the omega-limit sets of globally defined and relatively compact semiorbits: see Proposition II.2.1 of [32]. If $\mathcal{M}$ is a $\zeta$-invariant set, the restricted semiflow $\left(\mathcal{M}, \sigma, \mathbb{R}^{+}\right)$admits a continuous flow 
extension if there exists a continuous flow $(\mathcal{M}, \bar{\sigma}, \mathbb{R})$ such that $\bar{\sigma}(t, \omega)=\sigma(t, \omega)$ for all $t \in \mathbb{R}^{+}$and $\omega \in \mathcal{M}$. If $\mathcal{M}$ is locally compact, then the existence of a continuous flow extension is equivalent to the uniqueness of the complete orbit in $\mathcal{M}$ of each one of its points: see Theorem II.2.3 of [32].

Now let $\left(\Omega, \sigma, \mathbb{R}^{+}\right)$be a global continuous semiflow on a compact metric space $\Omega$, and let $X$ be a Banach space. We will represent $\omega \cdot t:=\sigma(t, \omega)$. A local semiflow $\left(\Omega \times X, \zeta, \mathbb{R}^{+}\right)$is a local skew-product semiflow with base $(\Omega, \sigma, \mathbb{R})$ and fiber $X$ if it takes the form

$$
\zeta: \mathcal{U} \subseteq \mathbb{R}^{+} \times \Omega \times X \rightarrow \Omega \times X, \quad(t, \omega, x) \mapsto(\omega \cdot t, u(t, \omega, x)) .
$$

It is frequently assumed that the base semiflow is in fact a flow. In this case, a compact set $\mathcal{K} \subset \Omega \times X$ is a copy of the base if it is $\zeta$-invariant and agrees with the graph of a continuous function $k: \Omega \rightarrow X$. Note that, in this case, $u(t, \omega, k(\omega))=$ $k(\omega \cdot t)$ for all $\omega \in \Omega$ and all $t \geq 0$.

The following definitions and properties refer to the case of a continuous skewproduct semiflow $\left(\Omega \times X, \zeta, \mathbb{R}^{+}\right)$. Recall that $d_{\Omega}$ is the distance in the metric space $\Omega$ and let $\|\cdot\|_{X}$ be the norm in the Banach space $X$. Given two subsets $\mathcal{C}_{1}$ and $\mathcal{C}_{2}$ of $\Omega \times X$, we denote the Hausdorff semidistance from $\mathcal{C}_{1}$ to $\mathcal{C}_{2}$ by

$$
\operatorname{dist}\left(\mathcal{C}_{1}, \mathcal{C}_{2}\right):=\sup _{\left(\omega_{1}, x_{1}\right) \in \mathcal{C}_{1}}\left(\inf _{\left(\omega_{2}, x_{2}\right) \in \mathcal{C}_{2}}\left(d_{\Omega}\left(\omega_{1}, \omega_{2}\right)+\left\|x_{1}-x_{2}\right\|_{X}\right)\right) .
$$

For further purposes we recall that the Hausdorff distance is defined by

$$
d_{\mathcal{H}}\left(\mathcal{C}_{1}, \mathcal{C}_{2}\right):=\max \left(\operatorname{dist}\left(\mathcal{C}_{1}, \mathcal{C}_{2}\right), \operatorname{dist}\left(\mathcal{C}_{2}, \mathcal{C}_{1}\right)\right)
$$

and that these definitions are valid if we substitute $\Omega \times X$ by any metric space.

Definition 2.1. A set $\mathcal{S} \subset \Omega \times X$ attracts a set $\mathcal{C} \subseteq \Omega \times X$ under $\zeta$ if $\zeta_{t}(\mathcal{C})$ is defined for all $t \geq 0$ and $\lim _{t \rightarrow \infty} \operatorname{dist}\left(\zeta_{t}(\mathcal{C}), \mathcal{S}\right)=0$. The semiflow $\zeta$ is bounded dissipative if there exists a bounded set $\mathcal{S}$ attracting all the bounded subsets of $\Omega \times X$ under $\zeta$. A set $\mathcal{A} \subset \Omega \times X$ is a global attractor if it is compact, $\zeta$-invariant, and it attracts every bounded subset of $\Omega \times X$ under $\zeta$. Finally, a set $\mathcal{S}$ is absorbing under $\zeta$ if, for any bounded set $\mathcal{B}$, there exists $t_{0}=t_{0}(\mathcal{S}, \mathcal{B})$ such that $\zeta_{t}(\mathcal{B}) \subseteq \mathcal{S}$ for all $t \geq t_{0}$.

Remarks 2.2. 1. It is immediate to observe that a semiflow $\zeta$ needs to be globally defined in order to be bounded dissipative, and that the existence of a bounded absorbing set ensures the bounded dissipativity of the semiflow and the boundedness of any semiorbit.

2. If a global attractor $\mathcal{A}$ exists, then it contains any other closed, bounded, and $\zeta$-invariant set $\mathcal{B}: 0=\lim _{t \rightarrow \infty} \operatorname{dist}\left(\zeta_{t}(\mathcal{B}), \mathcal{A}\right)=\lim _{t \rightarrow \infty} \operatorname{dist}(\mathcal{B}, \mathcal{A})$, which ensures that $\mathcal{B} \subseteq \mathcal{A}$. In particular, any $\zeta$-minimal set is contained in $\mathcal{A}$. A similar argument shows that $\mathcal{A}$ is contained in any closed bounded set that attracts all the bounded subsets of $\Omega \times X$ under $\zeta$. In particular, the attractor $\mathcal{A}$ is unique, and it is contained in any absorbing set.

3. Recall that the $\zeta$-invariance of the global attractor $\mathcal{A}$ means that any of its elements has a complete orbit in $\mathcal{A}$. If fact, a point $(\omega, x)$ belongs to $\mathcal{A}$ if and only it admits a complete orbit in $\Omega$ which is bounded: see e.g. Theorem 1.7 of [5].

The next concept will be fundamental in the proofs of the main results. 
Definition 2.3. Suppose that the Banach space $X$ is partially ordered. The semiflow $\left(\Omega \times X, \zeta, \mathbb{R}^{+}\right)$defined by (2.1) is monotone if, for all $\omega \in \Omega$ and all $x_{1}, x_{2} \in X$ satisfying $x_{1} \leq x_{2}$, it is $u\left(t, \omega, x_{1}\right) \leq u\left(t, \omega, x_{2}\right)$ for all $t \geq 0$ such that $\left(t, \omega, x_{1}\right)$ and $\left(t, \omega, x_{2}\right)$ belong to $\mathcal{U}$. In the case that the semiflow $\zeta$ is induced by a family of differential equations, the elements of the family are cooperative.

Let us now give the definition of uniform exponential stability, which refers to a compact set $\mathcal{K} \subset \Omega \times X$ projecting over the whole base; i.e., such that $\mathcal{K}_{\omega}:=$ $\{x \in X \mid(\omega, x) \in \mathcal{K}\}$ is nonempty for all $\omega \in \Omega$ (which is always the case if $\mathcal{K}$ is positively $\zeta$-invariant and $\Omega$ is minimal).

Definition 2.4. A positively $\zeta$-invariant compact set $\mathcal{K} \subset \Omega \times X$ projecting over the whole base is exponentially stable if there exist $\delta_{0}>0, k \geq 1$ and $\alpha>0$, such that, if $(\omega, \bar{x}) \in \mathcal{K}$ and $(\omega, x) \in \Omega \times X$ satisfy $\|x-\bar{x}\|_{X}<\delta_{0}$, then $u(t, \omega, x)$ is defined for $t \in[0, \infty)$ and $\|u(t, \omega, x)-u(t, \omega, \bar{x})\|_{X} \leq k e^{-\alpha t}\|x-\bar{x}\|_{X}$ for all $t \geq 0$. The restricted semiflow $\left(\mathcal{K}, \zeta, \mathbb{R}^{+}\right)$is said to be exponentially stable.

The last definition refers to the case of a linear skew-product semiflow. A global continuous skew-product semiflow $\zeta$ is linear if it takes the form

$$
\zeta: \mathbb{R}^{+} \times \Omega \times X \rightarrow \Omega \times X, \quad(t, \omega, x) \mapsto(\omega \cdot t, \phi(t, \omega) x),
$$

where $\phi(t, \omega): X \rightarrow X$ is a bounded linear operator. (See Remark 2.5 of [25] in order to see that the next definition makes sense also in the case that the base flow $\left(\Omega, \sigma, \mathbb{R}^{+}\right)$is a semiflow and not a flow. $)$

Definition 2.5. The upper Lyapunov exponent of the set $\Omega$ for the semiflow $\left(\Omega, \zeta, \mathbb{R}^{+}\right)$is

$$
\lambda_{\Omega}:=\sup _{\omega \in \Omega}\left(\sup _{x \in X, x \neq 0} \lambda_{s}^{+}(\omega, x)\right),
$$

where

$$
\lambda_{s}^{+}(\omega, x):=\limsup _{t \rightarrow \infty} \frac{1}{t} \ln \|\phi(t, \omega) x\|_{X} .
$$

Some notation used throughout the paper is now described. Given two Banach spaces $X$ and $Y$ with norms $\|\cdot\|_{X}$ and $\|\cdot\|_{Y}, \operatorname{Lin}(X, Y)$ represents the set of bounded linear maps $\phi: X \rightarrow Y$ equipped with the operator norm $\|\phi\|_{\operatorname{Lin}(X, Y)}=$ $\sup _{\|x\|_{X}=1}\|\phi(x)\|_{Y}$. Let us fix $r>0$. The set $C_{n}$ is the Banach space of continuous functions $C\left([-r, 0], \mathbb{R}^{n}\right)$ equipped with the norm $\|\psi\|_{C_{n}}:=\sup _{s \in[-r, 0]}|\psi(s)|$, where $|\cdot|$ represents the Euclidean norm in $\mathbb{R}^{n}$. The set $L^{\infty}$ is the space of Lebesguemeasurable functions $\psi:[-r, 0] \rightarrow \mathbb{R}^{n}$ which are essentially bounded, which means that there exists $k \geq 0$ such that the set $\{x \in[-r, 0]|| \psi(x) \mid>k\}$ has zero measure. The norm on $L^{\infty}$ is defined as the inferior of the set of real numbers $k \geq 0$ with the previous property and denoted by $\|\cdot\|_{L^{\infty}}$. The set $W_{n}^{1, \infty}$ is the Banach space of Lipschitz-continuous functions $\psi:[-r, 0] \rightarrow \mathbb{R}^{n}$ equipped with the norm

$$
\|\psi\|_{W_{n}^{1, \infty}}:=\max \left\{\|\psi\|_{C_{n}},\|\dot{\psi}\|_{L^{\infty}}\right\} .
$$

The subset of $W_{n}^{1, \infty}$ of the $C^{1}$-functions on $[-r, 0]$ will be denoted by $C_{n}^{1}$. Finally, given a continuous function $x:[-r, \gamma] \rightarrow \mathbb{R}^{n}$ for $\gamma>0$ and a time $t \in[0, \gamma]$, we denote by $x_{t} \in C_{n}$ the function defined by $x_{t}(s):=x(t+s)$ for $s \in[-r, 0]$. 
2.1. Some basic facts on state-dependent delay equations. Let $(\Omega, \sigma, \mathbb{R})$ be a continuous flow on a compact metric space, and let us consider the family of nonautonomous SDDEs

$$
\dot{y}(t)=F\left(\omega \cdot t, y(t), y\left(t-\tau\left(\omega \cdot t, y_{t}\right)\right)\right), \quad t \geq 0,
$$

for $\omega \in \Omega$, where $F$ and $\tau$ satisfy the following conditions:

H1 $F: \Omega \times \mathbb{R}^{n} \times \mathbb{R}^{n} \rightarrow \mathbb{R}^{n}$ is continuous, and its partial derivatives with respect to the second and third arguments exist and are continuous on $\Omega \times \mathbb{R}^{n} \times \mathbb{R}^{n}$.

H2 $\tau: \Omega \times C_{n} \rightarrow[0, r]$ is continuous and differentiable in the second argument, with $D_{2} \tau: \Omega \times C_{n} \rightarrow \operatorname{Lin}\left(C_{n}, \mathbb{R}\right)$ continuous.

We will use the notation $(2.5)_{\omega}$ to refer to the system of this family corresponding to the point $\omega$, and will proceed in an analogous way for the rest of the equations appearing in the paper.

The classical theory of finite-delay differential equations provides at least a solution $x(t)$ of a functional differential equation $x(t)=g\left(t, x_{t}\right)$ whenever $g$ is continuous: see e.g. Chapter 2 of [10]. But the uniqueness requires additional conditions on the Lipschitz behavior of $g$, which are not guaranteed by the conditions H1 and $\mathrm{H} 2$ : a simple adaptation to the case of finite delay of the the example of [37] described in Section 3.1 of [14] provides an equation with two solutions for the same continuous initial data. Theorem 1 of [12] shows that the uniqueness is indeed true under conditions $\mathrm{H} 1$ and $\mathrm{H} 2$ if the initial data is taken in $W_{n}^{1, \infty}$. The next result, strongly based on Theorem 1 of [12], is proved in Theorem 3.3 and Corollary 3.4 of [24] (which contain more information). It provides a semiflow $\left(\Omega \times W_{n}^{1, \infty}, \zeta, \mathbb{R}^{+}\right)$ whose global continuity cannot be ensured, but with strong continuity properties which make it a valuable tool for the use of the techniques of topological dynamics in the analysis of the long-term behavior of the solutions of (2.5).

Theorem 2.6. Suppose that conditions $\mathrm{H} 1$ and $\mathrm{H} 2$ hold. Then,

(i) for $\omega \in \Omega$ and $x \in W_{n}^{1, \infty}$, there exists a unique maximal solution $y(t, \omega, x)$ of the equation $(2.5)_{\omega}$ satisfying $y(s, \omega, x)=x(s)$ for $s \in[-r, 0]$, which is defined for $t \in\left[-r, \beta_{\omega, x}\right)$ with $0<\beta_{\omega, x} \leq \infty$. In particular, $y(t, \omega, x)$ is continuous on $\left[-r, \beta_{\omega, x}\right)$ and satisfies $(2.5)_{\omega}$ on $\left(0, \beta_{\omega, x}\right)$, and there exists the lateral derivative $\dot{y}\left(0^{+}, \omega, x\right)=F(\omega, y(0), y(-\tau(\omega, x))$.

Let us set

$$
\begin{aligned}
& \mathcal{U}_{n}:=\left\{(t, \omega, x) \mid(\omega, x) \in \Omega \times W_{n}^{1, \infty}, t \in\left[0, \beta_{\omega, x}\right)\right\} \subseteq \mathbb{R}^{+} \times \Omega \times W_{n}^{1, \infty}, \\
& \widetilde{\mathcal{U}}_{n}:=\left\{(t, \omega, x) \in \mathcal{U}_{n} \mid t \geq r\right\} \subset \mathbb{R}^{+} \times \Omega \times W_{n}^{1, \infty},
\end{aligned}
$$

provide them with the subspace topology, and define

$$
u(t, \omega, x)(s):=y(t+s, \omega, x)
$$

for every $(t, \omega, x) \in \mathcal{U}_{n}$ and $s \in[-r, 0]$, Then,

(ii) $u(t, \omega, x) \in W_{n}^{1, \infty}$ for all $t \in\left[0, \beta_{\omega, x}\right)$.

(iii) If $\sup _{t \in\left[0, \beta_{\omega, x}\right)}\|u(t, \omega, x)\|_{C}<\infty$ then $\beta_{\omega, x}=\infty$ and, in addition, the set $\{(\omega \cdot t, u(t, \omega, x)) \mid t \in[r, \infty)\} \subset \Omega \times W_{n}^{1, \infty}$ is relatively compact.

(iv) The set $\mathcal{U}_{n}$ is open in $\mathbb{R}^{+} \times \Omega \times W_{n}^{1, \infty}$ and the map

$$
\zeta: \mathcal{U}_{n} \subseteq \Omega \times W_{n}^{1, \infty} \rightarrow \Omega \times W_{n}^{1, \infty}, \quad(t, \omega, x) \mapsto(\omega \cdot t, u(t, \omega, x))
$$

defines a semiflow.

(v) The map $\mathcal{U} \rightarrow \Omega \times C_{n},(t, \omega, x) \mapsto(\omega \cdot t, u(t, \omega, x))$ is continuous. 
(vi) The map $\widetilde{\mathcal{U}}_{n} \rightarrow \Omega \times W_{n}^{1, \infty},(t, \omega, x) \mapsto(\omega \cdot t, u(t, \omega, x))$ is continuous.

(vii) Let us fix $\widetilde{t} \geq 0$ with $\left(\mathcal{U}_{n}\right)_{\tilde{t}}:=\{(\omega, x) \mid(\widetilde{t}, \omega, x) \in \mathcal{U}\}$ nonempty. Then the $\operatorname{map}\left(\mathcal{U}_{n}\right)_{\tilde{t}} \rightarrow \Omega \times W_{n}^{1, \infty},(\omega, x) \mapsto(\omega \cdot \widetilde{t}, u(\widetilde{t}, \omega, x))$ is continuous.

(viii) Let $\mathcal{K} \subset \Omega \times W_{n}^{1, \infty}$ be a positively $\zeta$-invariant compact set. Then the restriction of $\zeta$ to $\mathcal{K}$ defines a global continuous semiflow on $\mathcal{K}$.

(ix) The map $t \mapsto y(t, \omega, x)$ is $C^{1}$ on $\left[-r, \beta_{\omega, x}\right)$ if and only if $(\omega, x)$ belongs to

$$
\mathcal{C}_{n}^{0}:=\left\{(\omega, x) \in \Omega \times C_{n}^{1} \mid \dot{x}\left(0^{-}\right)=F(\omega, x(0), x(-\tau(\omega, x)))\right\},
$$

which is closed and positively $\zeta$-invariant, and $\zeta(t, \omega, x) \in \mathcal{C}_{n}^{0}$ if $(t, \omega, x) \in$ $\mathcal{U}_{n}$ and $t \geq r$. In addition, if $\mathcal{U}_{n}^{0}:=\left\{(t, \omega, x) \in \mathcal{U}_{n} \mid(\omega, x) \in \mathcal{C}_{n}^{0}\right\}$, then

$$
\zeta^{0}: \mathcal{U}_{n}^{0} \subseteq \mathbb{R}^{+} \times \mathcal{C}_{n}^{0} \rightarrow \mathcal{C}_{n}^{0}, \quad(t, \omega, x) \mapsto(\omega \cdot t, u(t, \omega, x))
$$

defines a continuous semiflow.

Remarks 2.7. 1. The definition of $\zeta$-invariant set is the same as in the case of a continuous semiflow. The assertions in Theorem 2.6(ix) make it easy to prove that any omega-limit set (and hence any minimal set) is $\zeta$-invariant, as in the continuous case. Also the concepts given in Definitions 2.1, 2.3 and 2.4 can be adapted to the case of our semiflow $\left(\Omega \times W_{n}^{1, \infty}, \zeta, \mathbb{R}^{+}\right)$, and the properties stated in Remarks 2.2 hold. In fact, the properties of regularity stated in the previous theorem will allow us to apply standard topological methods to the map $\zeta$ on $\Omega \times W_{n}^{1, \infty}$.

2. Theorem 2.6(ix) also ensures that any $\zeta$-invariant set, as omega-limit sets, minimal sets, and the global attractor (if it exists), is contained in the set $\mathcal{C}_{n}^{0}$ defined by (2.8), for which the restricted semiflow is continuous. And these sets are the key for the analysis of the long term dynamics.

Let us now consider the usual componentwise order in $\mathbb{R}^{n}:\left[\begin{array}{c}\alpha_{1} \\ \vdots \\ \alpha_{n}\end{array}\right] \leq\left[\begin{array}{c}\beta_{1} \\ \vdots \\ \beta_{n}\end{array}\right]$ if and only if $\alpha_{i} \leq \beta_{i}$ for $i=1, \ldots, n$. It is easy to check that the Euclidean norm in $\mathbb{R}^{n}$ is monotone: if $0 \leq \alpha \leq \beta$, then $|\alpha| \leq|\beta|$. It follows easily from here that a set $\mathcal{B} \subset \mathbb{R}^{n}$ is bounded if and only if there exist $\alpha$ and $\beta$ in $\mathbb{R}^{n}$ with $\alpha \leq x \leq \beta$ for all $x \in \mathcal{B}$. We also provide the Banach spaces $C_{n}$ and $W_{n}^{1, \infty}$ with the induced order

$$
x \leq y \quad \text { if and only if } \quad x(s) \leq y(s) \text { for all } s \in[-r, 0] .
$$

The relation $\geq$ is defined on $\mathbb{R}^{n}, C_{n}$ and $W_{n}^{1, \infty}$ in the obvious way.

Definition 2.8. Let $\tau: \Omega \times C_{n} \rightarrow[0, r]$ be a continuous function. We say that a function $F: \Omega \times \mathbb{R}^{n} \times \mathbb{R}^{n} \rightarrow \mathbb{R}^{n}$ satisfies the quasi-monotonicity condition for the delay function $\tau$ if it satisfies the following property:

$\mathbf{Q}$ If $x^{1}, x^{2} \in W_{n}^{1, \infty}$ satisfy $x^{1} \leq x^{2}$ and $x_{i}^{1}(0)=x_{i}^{2}(0)$ for an $i \in\{1, \ldots, n\}$, then $F_{i}\left(\omega, x^{1}(0), x^{1}\left(-\tau\left(\omega, x^{1}\right)\right)\right) \leq F_{i}\left(\omega, x^{2}(0), x^{2}\left(-\tau\left(\omega, x^{2}\right)\right)\right)$ for all $\omega \in$ $\Omega$.

The next comparison result adapts Theorem 1.1 of Chapter 5 of Smith [33] to SDDEs of the type (2.5), and can be proved in the same way: the required result on continuous dependence of the solutions with respect to parameters can be proved as point (v) of Theorem 3.2 of [24]. (To this regard, see also Remark 3.5 of [24]). In the statement of Theorem 2.9, the notation $u_{F}(t, \omega, x)$ corresponds to the function defined by (2.6), and $u_{G}(t, \omega, x)$ corresponds to the analogous one given by the new family $\dot{y}(t)=G\left(\omega \cdot t, y(t), y\left(t-\tau\left(\omega \cdot t, y_{t}\right)\right)\right)$ for $t \geq 0$ and $\omega \in \Omega$, with the same delay $\tau$ as the initial one, and with the same assumptions on $G$ as on $F$. 
Theorem 2.9. Let $\tau: \Omega \times C_{n} \rightarrow[0, r]$ satisfy $\mathrm{H} 2$, and let $F, G: \Omega \times \mathbb{R}^{n} \times \mathbb{R}^{n} \rightarrow \mathbb{R}^{n}$ satisfy H1. Suppose also that either $F$ or $G$ satisfies $\mathrm{Q}$ for $\tau$, and that $F\left(\omega, z^{1}, z^{2}\right) \leq$ $G\left(\omega, z^{1}, z^{2}\right)$ for all $\left(\omega, z^{1}, z^{2}\right) \in \Omega \times \mathbb{R}^{n} \times \mathbb{R}^{n}$. If $x$ and $\widetilde{x}$ in $W_{n}^{1, \infty}$ satisfy $x \leq \widetilde{x}$, then $u_{F}(t, \omega, x) \leq u_{G}(t, \omega, \widetilde{x})$ for all $t \geq 0$ in their common interval of definition.

An easy consequence follows (see Definition 2.3 and Remark 2.7.1):

Corollary 2.10. Suppose that the family (2.5) satisfies conditions $\mathrm{H} 1$ and $\mathrm{H} 2$, and that the function $F$ satisfies $\mathrm{Q}$ for the delay function $\tau$. Then the local semiflow $\left(\Omega \times W_{n}^{1, \infty}, \zeta, \mathbb{R}^{+}\right)$defined by $(2.7)$ is monotone.

\section{THE LONG-TERM DYNAMICS}

We analyze in this section the long-term dynamics of the solutions of a twodimensional system of nonautonomous SDDEs which models the so-called delayed cellular neural networks, namely

$$
\left\{\begin{array}{l}
\dot{y}_{1}(t)=-a_{1}^{0}(t) y_{1}(t)+b_{1}^{0}(t) f\left(y_{1}(t)\right)+c_{1}^{0}(t) g\left(y_{2}\left(t-\tau\left(y_{1}(t), y_{2}(t)\right)\right)\right)+I_{1}^{0}(t), \\
\dot{y}_{2}(t)=-a_{2}^{0}(t) y_{2}(t)+b_{2}^{0}(t) f\left(y_{1}\left(t-\tau\left(y_{1}(t), y_{2}(t)\right)\right)\right)+c_{2}^{0}(t) g\left(y_{2}(t)\right)+I_{2}^{0}(t) .
\end{array}\right.
$$

This system describes the dynamics of two groups of neurons and the relation of each group with itself, which we assume instantaneous, and with the other, which we assume delayed with state-dependent delay: the influence of the voltage of each neuron both in the synapse process and in the signal transmission justifies the statedependence of the delay in the model. The variables $y_{1}$ and $y_{2}$ describe an average value of the action potentials of the neurons in each group. The coefficients $a_{1}^{0}$ and $a_{2}^{0}$ are the decaying terms, the functions $b_{1}^{0}$ and $c_{2}^{0}$ are the synaptic coupling coefficients between different neurons of a same group, and the functions $c_{1}^{0}$ and $b_{2}^{0}$ are the synaptic coupling coefficients between neurons of different groups. These coefficients take average values of the (positive or negative) weights of the corresponding (excitatory or inhibitory) neuronal connections which, due to the plasticity of the network, may vary with respect to the time; and hence no assumption on their sign can be made. The coefficients $I_{1}^{0}$ and $I_{2}^{0}$ denote nonautonomous external inputs, and $f$ and $g$ are the bounded and increasing activation functions. The delay is given by the function $\tau$ which, as mentioned before, depends on the potentials (i.e. on the states) $y_{1}$ and $y_{2}$ : our model intends to be a more realistic approach to a simple biological method than the classical ones, described for instance in [38].

To state the precise conditions that we assume on this system, we recall that a function $f: \mathbb{R} \rightarrow \mathbb{R}$ is almost periodic if it is continuous and for any sequence $\left(s_{n}\right)$ of $\mathbb{R}$ there exist a subsequence $\left(t_{m}\right)$ and a continuous function $f^{*}: \mathbb{R} \rightarrow \mathbb{R}$ such that $\left(f_{t_{m}}\right)$ converges to $f^{*}$ uniformly in $\mathbb{R}$, where $f_{t}(s):=f(t+s)$.

Hypotheses 3.1. The coefficients $a_{1}^{0}, b_{1}^{0}, c_{1}^{0}, I_{1}^{0}, a_{2}^{0}, b_{2}^{0}, c_{2}^{0}$, and $I_{2}^{0}$ are almost periodic, with $a_{1}^{0}(t) \geq \delta$ and $a_{2}^{0}(t) \geq \delta$ for all $t \in \mathbb{R}$ for a constant $\delta>0$. In addition, the delay $\tau: \mathbb{R}^{2} \rightarrow[0, r]$ is a $C^{1}$ function, and the functions $f$ and $g$ belong to $C^{1}(\mathbb{R},[-1,1])$ and satisfy $\dot{f}, \dot{g}: \mathbb{R} \rightarrow[0,1]$.

The above is a representative simplified model of neuronal dynamics. Our purpose is to provide a dynamical theory for nonautonomous SDDEs, suitable to explain relevant features of the temporal evolution of the neuronal activity. Higher dimensional systems and more general expressions for the state-dependent delayed term can be considered in the model: the corresponding theory may be developed in a similar way. 
3.1. The hull construction and the definition of the semiflow. We will include the system (3.1) in a family of SDDEs such that each one of its systems is given by the evaluation of a continuous function along an orbit of a continuous flow on a compact metric space. The reason to perform this procedure is that, despite the nonautonomous character of our initial system, the solutions of the whole family will allow us to define a semiflow and hence to apply techniques from the topological dynamics. The conclusions are hence obtained for all the systems of the family, and can be particularized a posteriori for the initial system.

The procedure to do this is the classical Bebutov construction, which we explain now. We consider the function $l_{0}: \mathbb{R} \rightarrow \mathbb{R}^{8}$ given by $l_{0}:=\left(a_{1}^{0}, b_{1}^{0}, c_{1}^{0}, I_{1}^{0}, a_{2}^{0}, b_{2}^{0}, c_{2}^{0}, I_{2}^{0}\right)$. Let $\Omega$ be the hull of $l_{0}$, that is, the closure in the compact-open topology of the set of almost periodic maps $\left\{l_{t} \mid t \in \mathbb{R}\right\}$, with $l_{t}(s):=l_{0}(t+s)$ for $s \in \mathbb{R}$. It is a classical result that $\Omega$ is a compact metric space, and that the flow $\sigma$ defined on $\Omega$ by time-translation (i.e., $\sigma: \mathbb{R} \times \Omega \rightarrow \Omega,(t, \omega) \mapsto \omega \cdot t$ with $\omega \cdot t(s):=\omega(t+s)$ ) is continuous: see e.g. [9]. The hypotheses made on the coefficients of (3.1) ensure that $l_{0}$ is an almost periodic function, and thus $(\Omega, \sigma, \mathbb{R})$ is a minimal almost periodic flow: see Chapter VI of [31]. We represent by $\left(a_{1}, b_{1}, c_{1}, I_{1}, a_{2}, b_{2}, c_{2}, I_{2}\right)$ the (continuous) zero-evaluation operator on $\Omega$, which maps each $\omega \in \Omega$ to the vector $\omega(0)$ of $\mathbb{R}^{8}$. In this way, $a_{1}(\omega \cdot t)=\omega_{1}(t)$ (the fist component of $\omega(t) \in \mathbb{R}^{8}$ ), and the same happens with the remaining coefficients. Once this is done, we can consider the family of SDDEs

$$
\left\{\begin{aligned}
\dot{y}_{1}(t)= & -a_{1}(\omega \cdot t) y_{1}(t)+b_{1}(\omega \cdot t) f\left(y_{1}(t)\right) \\
& +c_{1}(\omega \cdot t) g\left(y_{2}\left(t-\tau\left(y_{1}(t), y_{2}(t)\right)\right)\right)+I_{1}(\omega \cdot t) \\
\dot{y_{2}}(t)= & -a_{2}(\omega \cdot t) y_{2}(t)+b_{2}(\omega \cdot t) f\left(y_{1}\left(t-\tau\left(y_{1}(t), y_{2}(t)\right)\right)\right) \\
& +c_{2}(\omega \cdot t) g\left(y_{2}(t)\right)+I_{2}(\omega \cdot t)
\end{aligned}\right.
$$

for $\omega \in \Omega$. The initial system (3.1) belongs to this family: it agrees with $(3.2)_{\omega_{0}}$ for $\omega_{0}:=l_{0}$. Note also that $a_{1}(\omega) \geq \delta$ and $a_{2}(\omega) \geq \delta$ for all $\omega \in \Omega$, with $\delta$ provided by Hypotheses 3.1 .

It is easy to check that the family (3.2) satisfies conditions $\mathrm{H} 1$ and $\mathrm{H} 2$ of Section 2.1 in the two-dimensional case. For each $(\omega, x) \in \Omega \times W_{2}^{1, \infty}$, we denote by $y(t, \omega, x)$ the solution of the equation $(3.2)_{\omega}$ with $y(s, \omega, x)=x(s)$ for $s \in[-r, 0]$, which is defined on a maximal interval $\left[-r, \beta_{\omega, x}\right)$. We also define $u(t, \omega, x)(s):=y(t+s, \omega, x)$ for $t \in\left[0, \beta_{\omega, x}\right)$ and $s \in[-r, 0]$, and consider the local semiflow $\left(\Omega \times W_{2}^{1, \infty}, \zeta, \mathbb{R}^{+}\right)$, where, as in $(2.7)$,

$$
\zeta: \mathcal{U}_{2} \subseteq \mathbb{R}^{+} \times \Omega \times W_{2}^{1, \infty} \rightarrow \Omega \times W_{2}^{1, \infty}, \quad(t, \omega, x) \mapsto(\omega \cdot t, u(t, \omega, x)) .
$$

It turns out that, in general, $\Omega$ is not a locally connected space: see [26]. Hence, it cannot be identified with a differentiable manifold, and this implies that $\mathcal{C}_{2}^{0}$ cannot be provided with the structure of a differentiable manifold, despite the fact that the fiber of $\mathcal{C}_{2}^{0}$ over each base point $\omega$ is a continuously differentiable submanifold of a Banach space varying with $\omega$ (see Proposition 3.4 of [36]).

3.2. Existence of the global attractor. In the rest of this section, we work under Hypotheses 3.1 , and $(\Omega, \sigma, \mathbb{R})$ and $\left(\Omega \times W_{2}^{1, \infty}, \zeta, \mathbb{R}^{+}\right)$are as above. Our first result establishes the existence of a global attractor, which is in addition connected. 
Theorem 3.2. Suppose that Hypotheses 3.1 hold. Then $\left(\Omega \times W_{2}^{1, \infty}, \zeta, \mathbb{R}^{+}\right)$is a bounded dissipative semiflow, and it admits a connected global attractor $\mathcal{A}$.

Proof. Let us rewrite the family $(3.2)$ as $\dot{y}_{i}(t)=F_{i}\left(\omega \cdot t, y(t), y\left(t-\tau\left(y_{t}\right)\right)\right.$ for $i=1,2$, and look for a constant $m$ such that, for all $\left(\omega, z_{1}, z_{2}\right) \in \Omega \times \mathbb{R}^{2} \times \mathbb{R}^{2}$ and $i=1,2$,

$$
-a_{i}(\omega) k_{i}-m \leq F_{i}\left(\omega, z_{1}, z_{2}\right) \leq-a_{i}(\omega) k_{i}+m .
$$

We consider two auxiliary families of (uncoupled) systems of linear ODEs,

$$
\left\{\begin{array} { l } 
{ \dot { y } _ { 1 } ( t ) = - a _ { 1 } ( \omega \cdot t ) y _ { 1 } ( t ) + m , } \\
{ \dot { y } _ { 2 } ( t ) = - a _ { 2 } ( \omega \cdot t ) y _ { 2 } ( t ) + m }
\end{array} \quad \text { and } \quad \left\{\begin{array}{l}
\dot{y}_{1}(t)=-a_{1}(\omega \cdot t) y_{1}(t)-m, \\
\dot{y}_{2}(t)=-a_{2}(\omega \cdot t) y_{2}(t)-m
\end{array}\right.\right.
$$

for $\omega \in \Omega$, and note that they satisfy property Q. Let us represent by $y^{+}(t, \omega, \alpha)=$ $\left[\begin{array}{l}y_{1}^{+}(t, \omega, \alpha) \\ y_{2}^{+}(t, \omega, \alpha)\end{array}\right]$ and $y^{-}(t, \omega, \alpha)=\left[\begin{array}{l}y_{1}^{-}(t, \omega, \alpha) \\ y_{2}^{-}(t, \omega, \alpha)\end{array}\right]$ the solutions of the left and right systems of $(3.5)_{\omega}$ with $y^{+}(0, \omega, \alpha)=y^{-}(0, \omega, \alpha)=\alpha \in \mathbb{R}^{2}$. It follows from (3.4) that the map $F=\left[\begin{array}{l}F_{1} \\ F_{2}\end{array}\right]: \Omega \times \mathbb{R}^{2} \times \mathbb{R}^{2} \rightarrow \mathbb{R}^{2}$ giving rise to (3.2) can be compared with those of the two families in (3.5) in the terms of Theorem 2.9, which consequently guarantees that

$$
y^{-}(t, \omega, x(0)) \leq y(t, \omega, x) \leq y^{+}(t, \omega, x(0))
$$

for $(\omega, x) \in \Omega \times W_{2}^{1, \infty}$ and $t \in\left[0, \beta_{\omega, x}\right)$. These inequalities and the global existence of $y^{ \pm}(t, \omega, x(0))$ combined with Theorem 2.6(iii) guarantee that $\beta_{\omega, x}=\infty$ for all $(\omega, x) \in \mathcal{C}_{2}^{0}$ : the semiflow $\left(\Omega \times W_{2}^{1, \infty}, \zeta, \mathbb{R}^{+}\right)$given by (3.3) is globally defined.

Using the fact that $a_{i}(\omega) \geq \delta>0$ for all $\omega \in \Omega$, we can get a $k_{*}>0$ such that

$$
-a_{i}(\omega) k+m<-1 \text { for all } \omega \in \Omega \text { and } k \geq k_{*},
$$

so that $-a_{i}(\omega) k-m>1$ for all $\omega \in \Omega$ and $k \leq-k_{*}$. The set $\mathcal{K}:=\left\{\left(\omega, z_{1}, z_{2}\right) \in\right.$ $\Omega \times \mathbb{R}^{2} \times \mathbb{R}^{2} \mid\left[\begin{array}{l}-k_{*} \\ -k_{*}\end{array}\right] \leq z_{i} \leq\left[\begin{array}{l}k_{*} \\ k_{*}\end{array}\right]$ for $\left.i=1,2\right\}$ is compact, so that there exists $d_{*}$ such that $-d_{*} \leq F_{i}\left(\omega, z_{1}, z_{2}\right) \leq d_{*}$ for all $\left(\omega, z_{1}, z_{2}\right) \in \mathcal{K}$ and $i=1,2$. We define

$$
\begin{aligned}
\mathcal{S}:=\left\{(\omega, x) \in \Omega \times W_{2}^{1, \infty} \mid\left[\begin{array}{l}
-k_{*} \\
-k_{*}
\end{array}\right] \leq x(s) \leq\left[\begin{array}{l}
k_{*} \\
k_{*}
\end{array}\right]\right. & \text { for all } s \in[-r, 0] \text { and } \\
{\left[\begin{array}{l}
-d_{*} \\
-d_{*}
\end{array}\right] \leq \dot{x}(s) \leq\left[\begin{array}{l}
d_{*} \\
d_{*}
\end{array}\right] } & \text { for a.a. } s \in[-r, 0]\} .
\end{aligned}
$$

The definition of $\mathcal{S}$ and the monotonicity of the Euclidean norm on $\mathbb{R}^{2}$ ensure that $\mathcal{S}$ is bounded and closed. We will check that it is $\zeta$-absorbing. It is easy to prove that for any $\alpha \in \mathbb{R},-k_{*} \leq y_{i}^{-}\left(t, \omega,\left[\begin{array}{c}-\alpha \\ -\alpha\end{array}\right]\right)$ and $y_{i}^{+}\left(t, \omega,\left[\begin{array}{c}\alpha \\ \alpha\end{array}\right]\right) \leq k_{*}$ for all $t \geq t_{\alpha}:=\max \left(0, \alpha-k_{*}\right), \omega \in \Omega$ and $i=1,2$ : just note that the inequalities persist to the right of any time $t$ at which they are satisfied; and if $\alpha>k^{*}$ use the bounds for the derivatives provided by (3.7).

Let $\mathcal{B} \subset \Omega \times W_{2}^{1, \infty}$ be an arbitrary bounded set, and let us choose $\alpha_{*}=\alpha_{*}(\mathcal{B}) \in \mathbb{R}$ such that $\left[\begin{array}{c}-\alpha_{*} \\ -\alpha_{*}\end{array}\right] \leq x(0) \leq\left[\begin{array}{c}\alpha_{*} \\ \alpha_{*}\end{array}\right]$ for all $(\omega, x) \in \mathcal{B}$. It is a well-known result that the uncoupled systems (3.5) define monotone global flows on $\Omega \times \mathbb{R}^{2}$ (see Definition 2.3 and Chapter 3 of [33]). These facts combined with (3.6) yield

$$
y^{-}\left(t, \omega,\left[\begin{array}{l}
-\alpha_{*} \\
-\alpha_{*}
\end{array}\right]\right) \leq y^{-}(t, \omega, x(0)) \leq y(t, \omega, x) \leq y^{+}(t, \omega, x(0)) \leq y^{+}\left(t, \omega,\left[\begin{array}{c}
\alpha_{*} \\
\alpha_{*}
\end{array}\right]\right)
$$

for all $(\omega, x) \in \mathcal{B}$ and $t \geq 0$. We define $t_{\mathcal{B}}:=t_{\alpha_{*}} \geq 0$ and deduce from the previous paragraph and (3.8) that $-k_{*} \leq y_{i}(t, \omega, x) \leq k_{*}$ for all $(\omega, x) \in \mathcal{B}$ whenever $t \geq t_{\mathcal{B}}$ and for $i=1,2$. This property and the definition of $d_{*}$ ensure that $-d_{*} \leq$ $\dot{y}_{i}(t, \omega, x) \leq d_{*}$ for all $(\omega, x) \in \mathcal{B}$ whenever $t \geq t_{\mathcal{B}}+r$ and $i=1,2$. That is, $(\omega \cdot t, u(t, \omega, x)) \in \mathcal{S}$ for all $(\omega, x) \in \mathcal{B}$ and $t \geq t_{\mathcal{B}}+2 r$; i.e., $\mathcal{S}$ is $\zeta$-absorbing. In particular, the semiflow $\left(\Omega \times W_{2}^{1, \infty}, \zeta, \mathbb{R}^{+}\right)$is bounded dissipative. 
Reviewing the argument, we observe that $t_{k_{*}}=0$, so that $t_{\mathcal{S}}=0$. It is easy to deduce that $\mathcal{S}$ is positively $\zeta$-invariant. Therefore, $\bigcup_{t \geq 0} \zeta_{t}(\mathcal{S})=\mathcal{S}$, so that this union is bounded. Clearly $\zeta_{2 r}(\mathcal{S})$ is absorbing under $\zeta$ and positively $\zeta$-invariant

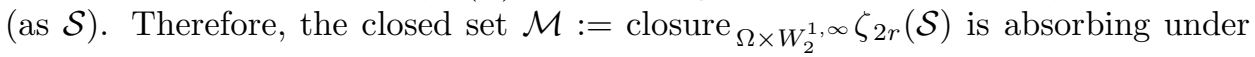
$\zeta$ and positively $\zeta$-invariant. (Here we make use of Theorem 2.6(vii).) These properties allow us to repeat the argument of the proof of Theorem 2.6(iii) (see Theorem 3.3(ix) of [24]) in order to check that $\mathcal{M}$ is compact.

We will now recall how to obtain the global attractor from $\mathcal{M}$, which is a standard procedure (despite the lack of global continuity of $\zeta$ ). Let us define $\mathcal{A}=\bigcap_{t \geq 0} \zeta_{t}(\mathcal{M})$, which is clearly a nonempty compact set, and it is positively $\zeta$-invariant (since $\mathcal{M}$ is). It is easy to check that $(\omega, x)$ belongs to $\mathcal{A}$ if and only if there exist sequences $\left(\left(\omega_{n}, x_{n}\right)\right)$ in $\mathcal{M}$ and $\left(t_{n}\right) \uparrow \infty$ such that $(\omega, x)=$ $\lim _{n \rightarrow \infty} \zeta\left(t_{n}, \omega_{n}, x_{n}\right)$, and to deduce that $\mathcal{A} \subseteq \zeta_{t}(\mathcal{A})$ for all $t \geq 0$. That is, $\mathcal{A}$ is $\zeta$-invariant. We will now check that $\mathcal{A}$ attracts any bounded set $\mathcal{B}$. Assume for contradiction that there exists $\varepsilon>0,\left(t_{n}\right) \uparrow \infty$, and a sequence $\left(\left(\omega_{n}, x_{n}\right)\right)$ in $\mathcal{B}$ such that $\varepsilon \leq \operatorname{dist}\left(\zeta\left(t_{n}, \omega_{n}, x_{n}\right), \mathcal{A}\right)$, where dist is defined by $(2.2)$ (for a singleton $\mathcal{C}_{1}$, and $\mathcal{C}_{2}=\mathcal{A}$ ). Since $\zeta\left(t_{n}, \omega_{n}, x_{n}\right)$ belongs to the (absorbing) set $\mathcal{M}$ for large enough $n$, there exist subsequences $\left(t_{m}\right)$ and $\left(\left(\omega_{m}, x_{m}\right)\right)$ and a point $(\omega, x)$ with $(\omega, x)=\lim _{m \rightarrow \infty} \zeta\left(t_{m}, \omega_{m}, x_{m}\right)$. But then $(\omega, x) \in \mathcal{A}$ and $\operatorname{dist}((\omega, x), \mathcal{A}) \geq \varepsilon$, which is impossible. All these properties show that $\mathcal{A}$ is a global attractor.

It remains to prove that $\mathcal{A}$ is connected. Note that it contains a minimal set, and hence it projects over the whole (minimal) base $\Omega$, which is connected. Let us call $\mathcal{A}^{2}:=\left\{x \in W_{2}^{1, \infty} \mid\right.$ there exists $\omega \in \Omega$ with $\left.(\omega, x) \in \mathcal{A}\right\}$, which is a compact set, and let $\widehat{\mathcal{A}}^{2}$ the closed convex hull of $\mathcal{A}^{2}$. Then the set $\Omega \times \widehat{\mathcal{A}}^{2}$ is compact and connected, and $\mathcal{A} \subseteq \widehat{\mathcal{A}}^{2}$. From this point we can follow the ideas of Lemma 2.4.1 of [11] in order to prove the connected character of $\mathcal{A}$. This completes the proof.

3.3. Global attractor and pullback attractors. It is well-know that the definition of the so-called process associated to a nonautonomous differential equation provides an approach to analyze its long term dynamics which is different from that of constructing the hull and the corresponding skew-product semiflow. Caraballo et al [4] combine both dynamical formalisms in the case of a nonautonomous ODE in order to describe the properties of the pullback attractor of the process from the structure of the global attractor for the skew-product semiflow. In what follows, we make a similar analysis for the case of our nonautonomous SDDEs: from those properties of the semiflow $\left(\Omega \times W_{2}^{1, \infty}, \zeta, \mathbb{R}^{+}\right)$which imply the existence of the global attractor $\mathcal{A}$, we deduce the existence of the pullback attractor for the initial process (and for all the processes associated to each one of the equations of the family), and determine its shape in terms of that of $\mathcal{A}$.

In what follows we assume that Hypotheses 3.1 hold. Let us fix, for the moment being, $\omega \in \Omega$, and define

$$
S_{\omega}(t, s): W_{2}^{1, \infty} \rightarrow W_{2}^{1, \infty}, x \mapsto S_{\omega}(t, s) x:=u(t-s, \omega \cdot s, x)
$$

for $t \geq s$. The cocycle equality $u(t+s, \omega, x)=u(t, \omega \cdot s, u(s, \omega, x)$ ) (which follows from the property (f2) for $\zeta$ ), the continuity of the base flow $(\Omega, \sigma, \mathbb{R})$, and Theorem 2.6(vi) and (vii) ensure that: $S_{\omega}(t, t)=\operatorname{Id}_{W_{2}^{1, \infty}}$ for all $t \geq 0 ; S_{\omega}(t, s)=$ $S_{\omega}(t, l) \circ S_{\omega}(l, s)$ whenever $t \geq l \geq s ;$ if

$$
\mathcal{F}_{\omega}:=\left\{(t, s, x) \in \mathbb{R} \times \mathbb{R} \times W_{2}^{1, \infty} \mid t-s \geq r\right\}
$$


then $S_{\omega}: \mathcal{F}_{\omega} \rightarrow W_{2}^{1, \infty},(t, s, x) \mapsto S_{\omega}(t, s) x$ is continuous (that is, the map $S_{\omega}$ defines a process, which can be not continuous due to the possible lack of continuity for $0 \leq t-s<r$ ); and

$$
S_{\omega}(t, s): W_{2}^{1, \infty} \rightarrow W_{2}^{1, \infty} \text { is continuous if } s \text { and } t \text { are fixed. }
$$

A continuous processes associated to the continuous semiflow $\left(\mathcal{C}_{2}^{0}, \zeta^{0}, \mathbb{R}^{+}\right)$given by (2.9) instead of $\left(\Omega \times W_{2}^{1, \infty}, \zeta, \mathbb{R}^{+}\right)$is defined in Section 3 of [36]. But in that case the domain and codomain of $S_{\omega}(t, s)$, given by sections of the set $\mathcal{C}_{2}^{0}$, vary with $\omega, t$ and $s$. Note that, for our process, the lack of continuity while $t-s \leq r$ is not relevant, since the long-term analysis is done for a fixed $t$ and $s \rightarrow-\infty$.

The distance between two subsets of $W_{n}^{1, \infty}$ is defined by the analogue of (2.2).

Definition 3.3. A time-dependent family $\left\{\mathcal{K}_{t} \mid t \in \mathbb{R}\right\}$ of compact subsets of $W_{2}^{1, \infty}$ pullback attracts bounded sets under $S_{\omega}$ if $\lim _{s \rightarrow-\infty} \operatorname{dist}\left(S_{\omega}(t, s) \mathcal{B}, \mathcal{K}_{t}\right)=0$ for all $t \in \mathbb{R}$ for every bounded set $\mathcal{B}$; and it is $S_{\omega}$-invariant if $S_{\omega}(t, s) \mathcal{K}_{s}=\mathcal{K}_{t}$ whenever $t \geq s$. An $S_{\omega}$-invariant family $\left\{\mathcal{K}_{t} \mid t \in \mathbb{R}\right\}$ of compact sets is a pullback attractor of $S_{\omega}$ if it pullback attracts bounded sets under $S_{\omega}$ and, in addition, it is the minimal $S_{\omega}$-invariant family of compact sets with this property (in the sense of set inclusion). And given a bounded set $\mathcal{B} \subset W_{2}^{1, \infty}$, its pullback omega-limit set in time $t \in \mathbb{R}$ is

$$
\mathcal{O}_{\omega}(\mathcal{B}, t):=\left\{x \in W_{2}^{1, \infty} \mid x=\lim _{n \rightarrow \infty} S_{\omega}\left(t, s_{n}\right) x_{n} \quad \text { for }\left(s_{n}\right) \downarrow-\infty \text { and }\left(x_{n}\right) \text { in } \mathcal{B}\right\} .
$$

Note that the minimality required in the definition of the pullback attractor implies its uniqueness, in the case of existence.

Proposition 3.4. Suppose that Hypotheses 3.1 hold. Let $\mathcal{B} \subset W_{2}^{1, \infty}$ be a bounded set. Then, for each $\omega \in \Omega$ and all $t \in \mathbb{R}$, the set $\mathcal{O}_{\omega}(\mathcal{B}, t)$ is nonempty and compact, and $\lim _{s \rightarrow-\infty} \operatorname{dist}\left(S_{\omega}(t, s) \mathcal{B}, \mathcal{O}_{\omega}(\mathcal{B}, t)\right)=0$. In addition, $S_{\omega}(t, s) \mathcal{O}_{\omega}(\mathcal{B}, s)=$ $\mathcal{O}_{\omega}(\mathcal{B}, t)$ for each $\omega \in \Omega$ whenever $t \geq s$.

Proof. Let us fix any $n \in \mathbb{N}$ and take the sets $\mathcal{S}$ and $\mathcal{M}$ defined in the proof of Theorem 3.2, so that $\mathcal{M}=\operatorname{closure}_{\Omega \times W_{2}^{1, \infty}} \zeta_{2 r}(\mathcal{S})$. Let us define $\mathcal{M}_{\Omega}:=\{x \in$ $W_{2}^{1, \infty} \mid$ there exists $\omega \in \Omega$ with $\left.(\omega, x) \in \mathcal{M}\right\}$, which is a compact subset of $W_{2}^{1, \infty}$. By repeating the ideas of the proof of Theorem 3.2 we show that, if $\mathcal{B} \subset W_{2}^{1, \infty}$ is bounded, then there exists $t_{\mathcal{B}}$ such that $S_{\omega}(t, s) \mathcal{B} \subseteq \mathcal{S}$ for $t-s \geq t_{\mathcal{B}}$ and hence that $S_{\omega}(t, s) \mathcal{B} \subseteq \mathcal{M}_{\Omega}$ for $t-s \geq t_{\mathcal{B}}+2 r$, for all $\omega \in \Omega$. In particular, $O_{\omega}(\mathcal{B}, t) \subseteq \mathcal{M}_{\Omega}$ for all $\omega \in \Omega$ and all $t \in \mathbb{R}$. In these conditions, we can repeat the proof of Lemma 2.7 of [5], whose conclusions are those of this proposition. Note that Lemma 2.7 of [5] relies on Lemma 2.4 of the same book, and that also this result can be adapted to our setting, due to (3.9).

Recall that $\mathcal{A}_{\omega}:=\left\{x \in W_{2}^{1, \infty} \mid(\omega, x) \in \mathcal{A}\right\}$ for any $\omega \in \Omega$.

Theorem 3.5. Suppose that Hypotheses 3.1 hold, and let $\mathcal{A}$ be the global attractor provided by Theorem 3.2. Then, for each $\omega \in \Omega$ and all $t \in \mathbb{R}$,

$$
\mathcal{A}_{\omega \cdot t}=\operatorname{closure}_{W_{2}^{1, \infty}}\left(\bigcup_{\mathcal{B} \subset W_{2}^{1, \infty}, \mathcal{B} \text { bounded }} \mathcal{O}_{\omega}(\mathcal{B}, t)\right) .
$$

In addition, $\left\{\mathcal{A}_{\omega \cdot t} \mid t \in \mathbb{R}\right\}$ is an $S_{\omega}$-invariant family of compact sets and it is the pullback attractor of the process $S_{\omega}$. 
Proof. We fix $\omega \in \Omega$ and define $\mathcal{K}_{t}:=\operatorname{closure}_{W_{2}^{1, \infty}}\left(\bigcup_{\mathcal{B} \subset W_{2}^{1, \infty}, \mathcal{B} \text { bounded }} \mathcal{O}_{\omega}(\mathcal{B}, t)\right)$. Let $\mathcal{M}_{\Omega}$ be defined as in the proof of Proposition 3.4. As said there, $\mathcal{O}_{\omega}(\mathcal{B}, t) \subseteq \mathcal{M}_{\Omega}$ for all $t \in \mathbb{R}$, so that $\mathcal{K}_{t} \subseteq \mathcal{M}_{\Omega}$ and hence it is a compact set. It follows from Proposition 3.4 that the family $\left\{\mathcal{K}_{t} \mid t \in \mathbb{R}\right\}$ of compact sets pullback-attracts bounded sets. In addition, $S_{\omega}(t, s) \mathcal{K}_{s}=\mathcal{K}_{t}$ for $t \geq s$ : this property follows from Proposition 3.4 and (3.9). Therefore, the family $\left\{\mathcal{K}_{t} \mid t \in \mathbb{R}\right\}$ is $S_{\omega}$-invariant.

In addition, if $\left\{\mathcal{P}_{t} \mid t \in \mathbb{R}\right\}$ is another $S_{\omega}$-invariant family of compact sets which attracts bounded sets, then $\mathcal{O}_{\omega}(\mathcal{B}, t) \subseteq \mathcal{P}_{t}$ for every bounded set $\mathcal{B} \subset W_{2}^{1, \infty}$. This property follows easily from the definition of $\mathcal{O}_{\omega}(\mathcal{B}, t)$ and a contradiction argument. Therefore, $\mathcal{K}_{t} \subseteq \mathcal{P}_{t}$. Consequently, $\left\{\mathcal{K}_{t} \mid t \in \mathbb{R}\right\}$ is the pullback attractor.

Let us finally check that $\mathcal{K}_{t}=\mathcal{A}_{\omega \cdot t}$ for all $t \in \mathbb{R}$. According to Remarks 2.2.3 and 2.7.1, a point $x \in W_{2}^{1, \infty}$ belongs to $\mathcal{A}_{\omega}$ if and only if $(\omega, x)$ admits a bounded complete orbit. Since $\mathcal{K}_{t} \subseteq \mathcal{M}_{\Omega}$ for all $t \in \mathbb{R}$, Theorem 1.17 of [5] (whose proof can be repeated without changes for our process) proves that this is the same condition required on the point $x \in W_{2}^{1, \infty}$ in order to belong to $\mathcal{K}_{0}$. Therefore, $\mathcal{K}_{0}=\mathcal{A}_{\omega}$. The same argument works for every $t \in \mathbb{R}$, and this completes the proof.

3.4. Properties of the global attractor. We continue working under Hypotheses 3.1, so that Theorem 3.2 ensures the existence of the global attractor $\mathcal{A}$ for the semiflow $\left(\Omega \times W_{2}^{1, \infty}, \mathbb{R}^{+}, \zeta\right)$. The restriction of $\zeta$ to the global attractor $\mathcal{A}$, which is continuous (see Theorem 2.6(viii)), determines the long-term behaviour of the bounded semiorbits of $\left(\Omega \times W_{2}^{1, \infty}, \zeta, \mathbb{R}^{+}\right)$. The following goal is to describe conditions which provide $\mathcal{A}$ with the simplest possible structure: that of a copy of the base, which means that $\mathcal{A}$ agrees with the graph of a continuous map $a: \Omega \rightarrow W_{2}^{1, \infty}$. This goal is achieved in Theorem 3.7, whose hypotheses consist of Hypotheses 3.1 together with the exponential stability of all the $\zeta$-minimal sets.

Remark 3.6. We recall two facts: the exponential stability of a $\zeta$-minimal set $\mathcal{M}$ is characterized in Section 5 of [25] by the negative character of its upper Lyapunov exponent with respect to $\zeta$ (see Definition 3.10 and Theorem 3.11 below); and all the $\zeta$-minimal sets are contained in the global attractor $\mathcal{A}$ (see Remarks 2.7.1 and 2.2.2). That is, the hypothesis of exponential stability of all the $\zeta$-minimal sets holds if the upper Lyapunov exponent of $\mathcal{A}$ is negative, which a priori seems to be a more restrictive property. But Theorem 3.7(iii) shows that both conditions are equivalent.

Theorem 3.7. Suppose that Hypotheses 3.1 hold, and that all the $\zeta$-minimal subsets of $\Omega \times W_{2}^{1, \infty}$ are exponentially stable. Let $\mathcal{A}$ be the global attractor provided by Theorem 3.2. Then,

(i) $\mathcal{A}$ is $\zeta$-minimal and the continuous semiflow $\left(\mathcal{A}, \zeta, \mathbb{R}^{+}\right)$admits a flow extension. In particular, $\mathcal{A}$ is the unique $\zeta$-minimal subset of $\Omega \times W_{2}^{1, \infty}$.

(ii) $\mathcal{A}$ is a copy of the base; i.e., there exists a continuous function a: $\Omega \rightarrow$ $W_{2}^{1, \infty}$ such that $\mathcal{A}=\{(\omega, a(\omega)) \mid \omega \in \Omega\}$.

(iii) There exist a constant $\beta>0$ and, for any $c>0$, a constant $k_{c} \geq 1$ such that, if $x \in W_{2}^{1, \infty}$ satisfies $\|x\|_{W_{2}^{1, \infty}} \leq c$, then

$$
\|u(t, \omega, x)-a(\omega \cdot t)\|_{W_{2}^{1, \infty}} \leq k_{c} e^{-\beta t}\|x-a(\omega)\|_{W_{2}^{1, \infty}} \quad \text { for all } t \geq 0 .
$$

Proof. (i) Recall that Hypotheses 3.1 include the minimality of $(\Omega, \sigma, \mathbb{R})$. According to Theorem 5.9 of [25], the exponential stability of all the $\zeta$-minimal sets (i.e., the 
strict negativeness of the upper Lyapunov exponent of all the $\zeta$-minimal sets), together with the fact that $\mathcal{A}$ is connected (see Theorem 3.2), ensures that $\mathcal{A}$ contains at most a $\zeta$-minimal set $\mathcal{M}$ which agrees with the omega-limit set of any of the elements of $\mathcal{A}$. In particular, the semiflow $\left(\Omega \times W_{2}^{1, \infty}, \zeta, \mathbb{R}^{+}\right)$admits just one $\zeta$-minimal set. In addition, according to Corollary 5.7 of [25], $\mathcal{M}$ is an $m$-cover of the base for an integer $m \geq 1$ admitting a flow extension. Therefore, (i) will be proved once shown that $\mathcal{A} \subseteq \mathcal{M}$.

Recall that the exponential stability of $\mathcal{M}$ means that there exist $\beta>0, k \geq 1$, and $\delta>0$ such that, if $(\omega, \bar{x}) \in \mathcal{M}$ and $(\omega, x) \in \Omega \times W_{2}^{1, \infty}$ satisfy $\|x-\bar{x}\|_{W_{2}^{1, \infty}}<\delta$, then the function $u(t, \omega, x)$ is defined for $t \in[0, \infty)$, and

$$
\|u(t, \omega, x)-u(t, \omega, \bar{x})\|_{W_{2}^{1, \infty}} \leq k e^{-\beta t}\|x-\bar{x}\|_{W_{2}^{1, \infty}} \text { for all } t \geq 0 .
$$

On the other hand, Corollary 5.7 of [25] (based on previous results of [29] and [28]) states that for each $\omega_{0} \in \Omega$ there exist a neighborhood $\mathcal{U}_{\omega_{0}} \subseteq \Omega$ of $\omega_{0}$ and $m$ continuous maps $a_{\omega_{0}}^{1}, \ldots, a_{\omega_{0}}^{m}: \mathcal{U}_{\omega_{0}} \rightarrow W_{2}^{1, \infty}$ such that

$$
\mathcal{M}_{\omega}:=\left\{x \in W_{2}^{1, \infty} \mid(\omega, x) \in \mathcal{M}\right\}=\left\{a_{\omega_{0}}^{1}(\omega), \ldots, a_{\omega_{0}}^{m}(\omega)\right\}
$$

for all $\omega \in \mathcal{U}_{\omega_{0}}$, with $a_{\omega_{0}}^{i}(\omega) \neq a_{\omega_{0}}^{j}(\omega)$ whenever $\omega \in \mathcal{U}_{\omega_{0}}$ and $i \neq j$.

We take $(\widetilde{\omega}, \widetilde{x}) \in \mathcal{A}$ and a negative semiorbit of it in $\mathcal{A}$ (see Remark 2.2.3), which we represent by $\left\{\left(\widetilde{\omega} \cdot t, \widetilde{x}_{t}\right) \mid t \leq 0\right\}$. The alpha-limit of $(\widetilde{\omega}, \widetilde{x})$ is a positively $\zeta$-invariant compact set, and hence it contains the unique $\zeta$-minimal set $\mathcal{M}$. Now we take $\left(\omega_{0}, x_{0}\right) \in \mathcal{M}$, assume without restriction that $x_{0}=a_{\omega_{0}}^{1}\left(\omega_{0}\right)$, and take a $\rho>0$ such that if $d_{\Omega}\left(\omega_{0}, \omega\right) \leq \rho$ then $\omega \in \mathcal{U}_{\omega_{0}}$ and $\| a_{\omega_{0}}^{1}\left(\omega_{0}\right)-$ $a_{\omega_{0}}^{1}(\omega) \|_{W_{2}^{1, \infty}} \leq \delta / 2$. Let us write $\left(\omega_{0}, x_{0}\right)=\lim _{n \rightarrow \infty}\left(\widetilde{\omega} \cdot\left(-t_{n}\right), \widetilde{x}_{-t_{n}}\right)$ for a suitable sequence $\left(t_{n}\right) \uparrow \infty$, and take $n_{0}$ such that, for all $n \geq n_{0}$, the following two properties hold: $d_{\Omega}\left(\omega_{0}, \widetilde{\omega} \cdot\left(-t_{n}\right)\right) \leq \rho$ and $\left\|x_{0}-\widetilde{x}_{-t_{n}}\right\|_{W_{2}^{1, \infty}}<\delta / 2$. It follows immediately that $\left\|\widetilde{x}_{-t_{n}}-a_{\omega_{0}}^{1}\left(\widetilde{\omega} \cdot\left(-t_{n}\right)\right)\right\|_{W_{2}^{1, \infty}} \leq\left\|\widetilde{x}_{-t_{n}}-a_{\omega_{0}}^{1}\left(\omega_{0}\right)\right\|_{W_{2}^{1, \infty}}+\| a_{\omega_{0}}^{1}\left(\omega_{0}\right)-$ $a_{\omega_{0}}^{1}\left(\widetilde{\omega} \cdot\left(-t_{n}\right)\right) \|_{W_{2}^{1, \infty}} \leq \delta$ for $n \geq n_{0}$. Consequently, by (3.10),

$$
\begin{aligned}
\| \widetilde{x} & -u\left(t_{n}, \widetilde{\omega} \cdot\left(-t_{n}\right), a_{\omega_{0}}^{1}\left(\widetilde{\omega} \cdot\left(-t_{n}\right)\right)\right) \|_{W_{2}^{1, \infty}} \\
& =\left\|u\left(t_{n}, \widetilde{\omega} \cdot\left(-t_{n}\right), \widetilde{x}_{-t_{n}}\right)-u\left(t_{n}, \widetilde{\omega} \cdot\left(-t_{n}\right), a_{\omega_{0}}^{1}\left(\widetilde{\omega} \cdot\left(-t_{n}\right)\right)\right)\right\|_{W_{2}^{1, \infty}} \leq k e^{-\beta t_{n}} \delta,
\end{aligned}
$$

which implies that

$$
(\widetilde{\omega}, \widetilde{x})=\lim _{n \rightarrow \infty}\left(\widetilde{\omega}, u\left(t_{n}, \widetilde{\omega} \cdot\left(-t_{n}\right), a_{\omega_{0}}^{1}\left(\widetilde{\omega} \cdot\left(-t_{n}\right)\right)\right)\right)=\lim _{n \rightarrow \infty} \zeta\left(t_{n}, \widetilde{\omega} \cdot\left(-t_{n}\right), a_{\omega_{0}}^{1}\left(\widetilde{\omega} \cdot\left(-t_{n}\right)\right)\right) .
$$

The points $\left(\widetilde{\omega} \cdot\left(-t_{n}\right), a_{\omega_{0}}^{1}\left(\widetilde{\omega} \cdot\left(-t_{n}\right)\right)\right.$ belong to $\mathcal{M}$, and consequently also the points $\zeta\left(t_{n}, \widetilde{\omega} \cdot\left(-t_{n}\right), a_{\omega_{0}}^{1}\left(\widetilde{\omega} \cdot\left(-t_{n}\right)\right)\right.$ belong to $\mathcal{M}$. Hence, $(\widetilde{\omega}, \widetilde{x}) \in \mathcal{M}$, and (i) is proved.

(ii) As said in the proof of (i), $\mathcal{A}=\mathcal{M}$ is an exponentially stable $m$-cover of the base. We will use the notation established in (3.11), having in mind that $\mathcal{A}_{\omega}=\mathcal{M}_{\omega}$, and choosing (without restriction) the neighborhood $\mathcal{U}_{\omega_{0}}$ of each $\omega_{0} \in \Omega$ to be compact. Let us assume for contradiction that $m \geq 2$. Then, there exists $d_{*}>0$ such that, for any $\omega \in \Omega$, any two points of $\mathcal{A}_{\omega}$ are at a distance greater than $d_{*}$. This assertion follows from the compactness of $\mathcal{U}_{\omega_{0}}$, which in turn ensures that $d_{\omega_{0}}:=\min _{1 \leq i<j \leq m, \omega \in \mathcal{U}}\left\|_{\omega_{0}}\right\| a_{\omega_{0}}^{i}(\omega)-a_{\omega_{0}}^{j}(\omega) \|_{W_{2}^{1, \infty}}>0$ for all $\omega_{0} \in \Omega$, and from the compactness of $\Omega$. The constant $d_{*}$ will play a key role in what follows.

Theorem 3.3 of [28] proves that the map $\omega \mapsto \mathcal{A}_{\omega}$ is continuous in the Hausdorff topology of the set of compact subsets of $W_{2}^{1, \infty}$. This fact ensures the existence of 
$\rho_{1}>0$ such that

$$
\text { if } d_{\Omega}\left(\omega_{1}, \omega_{2}\right) \leq \rho_{1} \quad \text { then } \max _{x_{1} \in \mathcal{A}_{\omega_{1}}}\left(\min _{x_{2} \in \mathcal{A}_{\omega_{2}}}\left\|x_{1}-x_{2}\right\|_{W_{2}^{1, \infty}}\right) \leq d_{*} / 6:
$$

see definition (2.3). In other words, if $d_{\Omega}\left(\omega_{1}, \omega_{2}\right) \leq \rho_{1}$ then for every $x_{1} \in \mathcal{A}_{\omega_{1}}$ there exists at least a point $x_{2} \in \mathcal{A}_{\omega_{2}}$ such that $\left\|x_{1}-x_{2}\right\|_{W_{2}^{1, \infty}} \leq d_{*} / 6$. We can assume without restriction that $\rho_{1} \leq d_{*} / 3$.

Let us fix $\omega_{0} \in \Omega$, choose $x_{0}$ and $x_{1}$ in $\mathcal{A}_{\omega_{0}}$ with $x_{0} \neq x_{1}$, and define $x_{\alpha}=$ $\alpha x_{1}+(1-\alpha) x_{0}$ for all $\alpha \in[0,1]$. Choose also a sequence $\left(t_{n}\right) \uparrow \infty$ such that $\lim _{n \rightarrow \infty} \omega_{0} \cdot t_{n}=\omega_{0}$, and choose $\rho_{2} \in\left(0, \rho_{1}\right]$ such that, if $d_{\Omega}\left(\omega, \omega_{0}\right) \leq \rho_{2}$, then $\omega \in \mathcal{U}_{\omega_{0}}$. Since $\mathcal{A}$ is the global attractor and the set $\mathcal{B}:=\left\{\left(\omega_{0}, x_{\alpha}\right) \mid \alpha \in[0,1]\right\}$ is bounded, there exists $n_{0}$ large enough to guarantee that $d_{\Omega}\left(\omega_{0} \cdot t^{*}, \omega_{0}\right) \leq \rho_{2} / 2$ and $\operatorname{dist}\left(\zeta_{t^{*}}(\mathcal{B}), \mathcal{A}\right)<\rho_{2} / 2$ for $t^{*}=t_{n_{0}}>0$. The definition of dist (see $(2.2)$ ) ensures that, for each $\alpha \in[0,1]$ there exists $\left(\omega_{\alpha}, x_{\alpha}^{*}\right) \in \mathcal{A}$ with $d_{\Omega}\left(\omega_{0} \cdot t^{*}, \omega_{\alpha}\right)+$ $\left\|u\left(t^{*}, \omega_{0}, x_{\alpha}\right)-x_{\alpha}^{*}\right\|_{W_{2}^{1, \infty}} \leq \rho_{2} / 2$. In particular, for each $\alpha \in[0,1], d_{\Omega}\left(\omega_{0}, \omega_{\alpha}\right) \leq \rho_{2}$, and hence there exists $j(\alpha) \in\{1, \ldots, m\}$ such that $x_{\alpha}^{*}=a_{\omega_{0}}^{j(\alpha)}\left(\omega_{\alpha}\right)$; and, since $d_{\Omega}\left(\omega_{0} \cdot t^{*}, \omega_{\alpha}\right) \leq \rho_{1}$, we deduce from (3.12) that there exists $i(\alpha) \in\{1, \ldots, m\}$ with $\left\|a_{\omega_{0}}^{i(\alpha)}\left(\omega_{0} \cdot t^{*}\right)-a_{\omega_{0}}^{j(\alpha)}\left(\omega_{\alpha}\right)\right\|_{W_{2}^{1, \infty}} \leq d_{*} / 6$. Therefore,

$$
\begin{aligned}
\left\|u\left(t^{*}, \omega_{0}, x_{\alpha}\right)-a_{\omega_{0}}^{i(\alpha)}\left(\omega_{0} \cdot t^{*}\right)\right\|_{W_{2}^{1, \infty}} \leq\left\|u\left(t^{*}, \omega_{0}, x_{\alpha}\right)-a_{\omega_{0}}^{j(\alpha)}\left(\omega_{\alpha}\right)\right\|_{W_{2}^{1, \infty}} \\
+\left\|a_{\omega_{0}}^{j(\alpha)}\left(\omega_{\alpha}\right)-a_{\omega_{0}}^{i(\alpha)}\left(\omega_{0} \cdot t^{*}\right)\right\|_{W_{2}^{1, \infty}} \leq \frac{\rho_{2}}{2}+\frac{d_{*}}{6} \leq \frac{d_{*}}{3}
\end{aligned}
$$

for all $\alpha \in[0,1]$. Note also that $i(\alpha)$ is the unique index satisfying the previous bound: the existence of two of them would provide two points on $\mathcal{A}_{\omega_{0} \cdot t^{*}}$ at a distance no greater than $2 d_{*} / 3$, which is impossible. In particular,

$$
\left(\omega_{0} \cdot t^{*}, a_{\omega_{0}}^{i(0)}\left(\omega_{0} \cdot t^{*}\right)\right)=\zeta\left(t^{*}, \omega_{0}, x_{0}\right) \text { and }\left(\omega_{0} \cdot t^{*}, a_{\omega_{0}}^{i(1)}\left(\omega_{0} \cdot t^{*}\right)\right)=\zeta\left(t^{*}, \omega_{0}, x_{1}\right),
$$

since all these points belong to the $\zeta$-invariant set $\mathcal{A}$.

The next goal is to check that the map $\alpha \mapsto i(\alpha)$ is constant on [0,1], which is the same as saying that it is locally constant. Note that there is no restriction in assuming that $t^{*} \geq r$. We fix $\alpha_{0} \in[0,1]$ and use the continuity of the semiflow $\zeta$ guaranteed by Theorem 2.6(vi) to find $\varepsilon>0$ such that, if $\alpha \in\left(\alpha_{0}-\varepsilon, \alpha_{0}+\right.$ $\varepsilon) \cap[0,1]$, then $\left\|u\left(t^{*}, \omega, x_{\alpha_{0}}\right)-u\left(t^{*}, \omega, x_{\alpha}\right)\right\|_{W_{2}^{1, \infty}}<d_{*} / 3$. This inequality, together with (3.13), means that $\left\|a_{\omega_{0}}^{i\left(\alpha_{0}\right)}\left(\omega_{0} \cdot t^{*}\right)-a_{\omega_{0}}^{i(\alpha)}\left(\omega_{0} \cdot t^{*}\right)\right\|_{W_{2}^{1, \infty}}<d_{*}$, and the assertion follows once more from the definition of $d_{*}$.

The last property and (3.14) prove that $\zeta\left(t^{*}, \omega_{0}, x_{0}\right)=\zeta\left(t^{*}, \omega_{0}, x_{1}\right)$, which is impossible, since $\zeta$ defines a flow on $\mathcal{A}$ and $x_{0} \neq x_{1}$. This contradiction proves that $m=1$. The existence of the continuous map $a: \Omega \rightarrow W_{2}^{1, \infty}$ such that $\mathcal{A}=$ $\{(\omega, a(\omega)) \mid \omega \in \Omega\}$ is a trivial consequence of this fact (recall that $\mathcal{A}$ is closed), and the proof of (ii) is complete.

(iii) Note that $u(t, \omega, a(\omega))=a(\omega \cdot t)$ for all $t \in \mathbb{R}$ and $\omega \in \Omega$. For any $c>0$, we define $\mathcal{B}_{c}:=\left\{x \in W_{2}^{1, \infty} \mid\|x\|_{W_{2}^{1, \infty}} \leq c\right\}$, which is a bounded subset of $W_{2}^{1, \infty}$. Since $\mathcal{A}$ is the global attractor, there exists $t_{c}$ such that $\operatorname{dist}\left(\zeta_{t_{c}}\left(\Omega \times \mathcal{B}_{c}\right), \mathcal{A}\right)<\delta$, where $\delta$ is determined by (3.10). Therefore, if $x \in \mathcal{B}_{c}$,

$$
\begin{aligned}
\|u(t, \omega, x)-a(\omega \cdot t)\|_{W_{2}^{1, \infty}} & =\left\|u\left(t-t_{c}, \omega \cdot t_{c}, u\left(t_{c}, \omega, x\right)\right)-u\left(t-t_{c}, \omega \cdot t_{c}, a\left(\omega \cdot t_{c}\right)\right)\right\|_{W_{2}^{1, \infty}} \\
& \leq k e^{-\beta\left(t-t_{c}\right)}\left\|u\left(t_{c}, \omega, x\right)-a\left(\omega \cdot t_{c}\right)\right\|_{W_{2}^{1, \infty}}
\end{aligned}
$$


for all $t \geq t_{c}$. The properties guaranteed by Hypotheses 3.1 ensure the existence of $l_{c} \geq 1$ such that $\|u(t, \omega, x)-a(\omega \cdot t)\|_{W_{2}^{1, \infty}} \leq l_{c}\|x-a(\omega)\|_{W_{2}^{1, \infty}}$ whenever $(\omega, x) \in$ $\Omega \times \mathcal{B}_{c}$ for all $t \in\left[0, t_{c}\right]$ : see Theorem 3.6 of [24]. The assertion in (iii) is satisfied by $k_{c}:=k l_{c} e^{\beta t_{c}} \geq l_{c}$.

Remark 3.8. Note that, since the orbits of $(\Omega, \sigma, \mathbb{R})$ are almost periodic (and with the same frequency module: see e.g. [17]), the existence of a copy of the base ensures the existence of at least one almost periodic solution for each one of the systems of the family, including of course the initial one (3.1). If, in addition, the copy of the base is a global attractor, then this almost periodic solution is unique (this follows, for example, from Remark 2.2.2); and, if this attractor is exponentially stable, so is the almost periodic solution. Altogether, these properties read as: if the hypotheses of Theorem 3.7 hold, then the initial system (3.1) has a unique almost periodic solution, which in addition is exponentially stable.

3.5. Bounding the upper Lyapunov exponents. As we advanced in Remark 3.6 (and will formulate explicitly in Theorem 3.11), the hypotheses regarding exponential stability of Theorem 3.7 hold if and only if the upper Lyapunov exponents of the $\zeta$-minimal sets are negative (in which case the unique minimal set is the global attractor $\mathcal{A}$ ). So that the obvious question is how to obtain these exponents, or at least how to bound them. The rest of this section is focussed on this problem.

Let us first recall the definition of the upper Lyapunov exponent of a $\zeta$-invariant compact subset $\mathcal{K} \subset \Omega \times W_{2}^{1, \infty}$. The set $\mathcal{K}$ could be, for instance, any $\zeta$-minimal set or omega-limit set, and either is contained in the global attractor $\mathcal{A}$ provided by Theorem 3.2 under Hypotheses 3.1, or it is the set $\mathcal{A}$ itself: see Remarks 2.2.1 and 2.7.1. According to Theorem 2.6(viii), $\left(\mathcal{K}, \zeta, \mathbb{R}^{+}\right)$is a continuous semiflow. We represent the elements of $\mathcal{A}$ by $\widehat{\omega}=(\omega, x)$ with $x=\left[\begin{array}{l}x_{1} \\ x_{2}\end{array}\right]$, and note that Remark 2.7.2 ensures that $x \in C_{2}^{1} \subset W_{2}^{1, \infty}$. We write $\widehat{\omega} \cdot t=\zeta(t, \widehat{\omega})$ for $\widehat{\omega} \in \mathcal{A}$ and $t \geq 0$, and consider the family of linear variational equations

$$
\left\{\begin{array}{l}
\dot{z}_{1}(t)=A_{1}(\widehat{\omega} \cdot t) z_{1}(t)+B_{1}(\widehat{\omega} \cdot t) z_{2}(t)+C_{1}(\widehat{\omega} \cdot t) z_{2}(t-\widehat{\tau}(\widehat{\omega} \cdot t)) \\
\dot{z}_{2}(t)=A_{2}(\widehat{\omega} \cdot t) z_{2}(t)+B_{2}(\widehat{\omega} \cdot t) z_{1}(t)+C_{2}(\widehat{\omega} \cdot t) z_{1}(t-\widehat{\tau}(\widehat{\omega} \cdot t))
\end{array}\right.
$$

for $\widehat{\omega}=(\omega, x) \in \mathcal{A}$, with $\widehat{\tau}: \mathcal{K} \rightarrow[0, r], \widehat{\omega} \mapsto \tau\left(x_{1}(0), x_{2}(0)\right)$, and where $A_{1}, B_{1}, C_{1}$, $A_{2}, B_{2}, C_{2}: \mathcal{A} \rightarrow \mathbb{R}$ are defined by

$$
\begin{aligned}
A_{1}(\widehat{\omega}):= & -a_{1}(\omega)+b_{1}(\omega) \dot{f}\left(x_{1}(0)\right) \\
& -c_{1}(\omega) \dot{g}\left(x_{2}\left(-\tau\left(x_{1}(0), x_{2}(0)\right)\right)\right) \dot{x_{2}}\left(-\tau\left(x_{1}(0), x_{2}(0)\right)\right) \frac{d \tau}{d y_{1}}\left(x_{1}(0), x_{2}(0)\right), \\
B_{1}(\widehat{\omega}):= & -c_{1}(\omega) \dot{g}\left(x_{2}\left(-\tau\left(x_{1}(0), x_{2}(0)\right)\right)\right) \dot{x_{2}}\left(-\tau\left(x_{1}(0), x_{2}(0)\right)\right) \frac{d \tau}{d y_{2}}\left(x_{1}(0), x_{2}(0)\right), \\
C_{1}(\widehat{\omega}):= & c_{1}(\omega) \dot{g}\left(x_{2}\left(-\tau\left(x_{1}(0), x_{2}(0)\right)\right)\right), \\
A_{2}(\widehat{\omega}):= & -a_{2}(\omega)+c_{2}(\omega) \dot{g}\left(x_{2}(0)\right) \\
& -b_{2}(\omega) \dot{f}\left(x_{1}\left(-\tau\left(x_{1}(0), x_{2}(0)\right)\right)\right) \dot{x_{1}}\left(-\tau\left(x_{1}(0) x_{2}(0)\right)\right) \frac{d \tau}{d y_{2}}\left(x_{1}(0), x_{2}(0)\right), \\
B_{2}(\widehat{\omega}):= & -b_{2}(\omega) \dot{f}\left(x_{1}\left(-\tau\left(x_{1}(0), x_{2}(0)\right)\right)\right) \dot{x_{1}}\left(-\tau\left(x_{1}(0), x_{2}(0)\right)\right) \frac{d \tau}{d y_{1}}\left(x_{1}(0), x_{2}(0)\right), \\
C_{2}(\widehat{\omega}):= & b_{2}(\omega) \dot{f}\left(x_{1}\left(-\tau\left(x_{1}(0), x_{2}(0)\right)\right)\right) .
\end{aligned}
$$


As is explained in Sections 4 of [24] and 3 of [25], the family (3.15) induces two globally defined linear skew-product semiflows, namely

$$
\begin{array}{lll}
\zeta_{L}: \mathbb{R}^{+} \times \mathcal{A} \times W_{2}^{1, \infty} \rightarrow \mathcal{A} \times W_{2}^{1, \infty}, & (t, \widehat{\omega}, v) \mapsto(\widehat{\omega} \cdot t, w(t, \widehat{\omega}, v)), \\
\widetilde{\zeta}_{L}: \mathbb{R}^{+} \times \mathcal{A} \times C_{2} \rightarrow \mathcal{A} \times C_{2}, & (t, \widehat{\omega}, v) \mapsto(\widehat{\omega} \cdot t, w(t, \widehat{\omega}, v)) .
\end{array}
$$

In both cases, $w(t, \widehat{\omega}, v)(s):=z(t+s, \widehat{\omega}, v)$ for $s \in[-r, 0]$, where $z(t, \widehat{\omega}, v)$ represents the solution of the system (3.15) corresponding to $\widehat{\omega} \in \mathcal{A}$ with $z(s, \widehat{\omega}, v)=v(s)$ for $s \in[-r, 0]$. (And, in fact, $w(t, \omega, x, v)$ determines the derivative with respect to the initial condition $x$ in the direction of the vector $v$ of the solution $u(t, \omega, x)$ lying in the set $\mathcal{A}$; that is, $w(t, \omega, x, v)=u_{x}(t, \omega, x) v=\lim _{\varepsilon \rightarrow 0}(u(t, \omega, x+\varepsilon v)-u(t, \omega, x)) / \varepsilon$. $)$ Note that the difference between $\zeta_{L}$ and $\widetilde{\zeta}_{L}$ relies on their domains of definition: the restriction of $\widetilde{\zeta}_{L}$ to $\mathcal{A} \times W_{2}^{1, \infty}$ agrees with $\zeta_{L}$. In fact, $\left(\mathcal{A} \times C_{2}, \widetilde{\zeta}_{L}, \mathbb{R}^{+}\right)$is a continuous semiflow, as Corollary 4.3 of $[24]$ proves; while $\left(\mathcal{A} \times W_{2}^{1, \infty}, \zeta_{L}, \mathbb{R}^{+}\right)$ satisfies similar properties to those described in Theorem 2.6, which are also detailed in Corollary 4.3 of [24].

Let $\mathcal{K}$ be a $\zeta$-invariant compact set. Then $\zeta_{L}$ and $\widetilde{\zeta}_{L}$ can be restricted to $\mathcal{K} \times$ $W_{2}^{1, \infty}$ and $\mathcal{K} \times C_{2}$. We represent by $\lambda_{\mathcal{K}}$ and $\widetilde{\lambda}_{\mathcal{K}}$ the upper Lyapunov exponents of $\mathcal{K}$ for the semiflows $\left(\mathcal{K} \times W_{2}^{1, \infty}, \zeta_{L}, \mathbb{R}^{+}\right)$and $\left(\mathcal{K} \times C_{2}, \widetilde{\zeta}_{L}, \mathbb{R}^{+}\right)$, respectively (see Definition 2.5, and note that it can be adapted without changes to the case of the semiflow $\left(\mathcal{A} \times W_{2}^{1, \infty}, \zeta_{L}, \mathbb{R}^{+}\right)$, as explained in Remark 3.9 of [25]). The following property, fundamental for our purposes, is proved in Theorem 3.10 of [25].

Theorem 3.9. Suppose that Hypotheses 3.1 hold. Then, $\lambda_{\mathcal{K}}=\widetilde{\lambda}_{\mathcal{K}}$.

Definition 3.10. Suppose that Hypotheses 3.1 hold. The upper Lyapunov exponent of the set $\mathcal{K}$ for the semiflow $\left(\mathcal{K}, \zeta, \mathbb{R}^{+}\right)$is $\lambda_{\mathcal{K}}=\widetilde{\lambda}_{\mathcal{K}}$.

The next result, previously mentioned, is part of Theorem 5.2 of [25].

Theorem 3.11. Suppose that Hypotheses 3.1 hold, and let $\mathcal{M} \subset \Omega \times W_{2}^{1, \infty}$ be a minimal set. Then, $\mathcal{M}$ is exponentially stable if and only if $\lambda_{\mathcal{M}}<0$.

The difficulty to estimate the value of the upper-Lyapunov exponent is obvious in the case that we are considering: the equations of the family (3.15) are written in terms of the semiorbits of $\left(\mathcal{A}, \zeta, \mathbb{R}^{+}\right)$, which in general are unknown. So that our goal is to bound $\lambda_{\mathcal{K}}$ (for all $\mathcal{K}$ as described before) by a quantity which is easier to estimate. Our method is based on results of comparison of solutions applied to the equations of the family (3.15) and those of two families of cooperative linear systems of FDEs (see Definition 2.3): (3.17) and (3.18). The advantages of estimating the upper Lyapunov exponents for these families are explained in Remark 3.14.

So, we first consider the family of FDEs

$$
\left\{\begin{array}{l}
\dot{z}_{1}(t)=A_{1}(\widehat{\omega} \cdot t) z_{1}(t)+\left|B_{1}(\widehat{\omega} \cdot t)\right| z_{2}(t)+\left|C_{1}(\widehat{\omega} \cdot t)\right| z_{2}(t-\widehat{\tau}(\widehat{\omega} \cdot t)), \\
\dot{z}_{2}(t)=A_{2}(\widehat{\omega} \cdot t) z_{2}(t)+\left|B_{2}(\widehat{\omega} \cdot t)\right| z_{1}(t)+\left|C_{2}(\widehat{\omega} \cdot t)\right| z_{1}(t-\widehat{\tau}(\widehat{\omega} \cdot t))
\end{array}\right.
$$

for $\widehat{\omega} \in \mathcal{A}$, which we write for short as $\dot{z}(t)=\bar{L}(\widehat{\omega} \cdot t) z_{t}$. For each $v \in C_{2}$, we denote by $\bar{z}(t, \widehat{\omega}, v)$ the solution of (3.17) satisfying $\bar{z}(s, \widehat{\omega}, v)=v(s)$ for $s \in[-r, 0]$, and define $\bar{w}(t, \widehat{\omega}, v)(s)=\bar{z}(t+s, \widehat{\omega}, v)$ for $s \in[-r, 0]$. Then the map

$$
\bar{\zeta}_{L}: \mathbb{R}^{+} \times \mathcal{A} \times C_{2} \rightarrow \mathcal{A} \times C_{2}, \quad(t, \widehat{\omega}, v) \mapsto(\widehat{\omega} \cdot t, \bar{w}(t, \widehat{\omega}, v))
$$


defines a linear skew-product semiflow on $\mathcal{A} \times C_{2}$. It is easy to check that, for all $\widehat{\omega} \in \mathcal{A}$, the vector field $(t, z) \mapsto \bar{L}(\widehat{\omega} \cdot t) z_{t}$ satisfies the quasimonotonicity condition on $\mathbb{R} \times C_{2}$ described in Chapter 5 of [33] (which is the same as our condition $\mathrm{Q}$ but for $x^{1}$ and $x^{2}$ in $C_{2}$ ). Therefore, Theorem 1.1 of the same chapter shows that the semiflow $\left(\mathcal{A} \times C_{2}, \bar{\zeta}_{L}, \mathbb{R}^{+}\right)$is monotone (see Definition 2.3). Clearly, $\bar{\zeta}_{L}$ can be restricted to $\mathcal{K} \times C_{2}$. We represent by $\bar{\lambda}_{\mathcal{K}}$ the upper Lyapunov exponent of $\mathcal{K}$ for the semiflow $\left(\mathcal{K} \times C_{2}, \bar{\zeta}_{L}, \mathbb{R}^{+}\right)$.

The following notation will be used from now on: given a two-dimensional real vector or function $x=\left[\begin{array}{c}x_{1} \\ x_{2}\end{array}\right]$, we denote $|x|_{m}=\left[\begin{array}{l}\left|x_{1}\right| \\ \left|x_{2}\right|\end{array}\right]$. Note that $|v|_{m} \in C_{2}$ if $v \in C_{2}$, and that $\|v\|_{C_{2}}=\left\||v|_{m}\right\|_{C_{2}}$.

Theorem 3.12. Suppose that Hypotheses 3.1 hold. Then,

(i) $|z(t, \widehat{\omega}, v)|_{m} \leq \bar{z}\left(t, \widehat{\omega},|v|_{m}\right)$ for all $v \in C_{2}$.

(ii) If $\mathcal{M} \subset \Omega \times W_{2}^{1, \infty}$ is a $\zeta$-minimal set, then $\lambda_{\mathcal{M}} \leq \bar{\lambda}_{\mathcal{M}}$.

(iii) If $\bar{\lambda}_{\mathcal{M}}<0$ for all the $\zeta$-minimal sets $\mathcal{M} \subset \Omega \times W_{2}^{1, \infty}$, then all the conclusions of Theorem 3.7 hold.

Proof. Statement (i) is proved in Lemma 4.2 of Novo et al. [27], and ensures that

$$
\limsup _{t \rightarrow \infty} \frac{1}{t} \ln \left\|z_{t}(\widehat{\omega}, v)\right\|_{C_{2}} \leq \limsup _{t \rightarrow \infty} \frac{1}{t} \ln \left\|\bar{z}_{t}\left(\widehat{\omega},|v|_{m}\right)\right\|_{C_{2}} .
$$

This inequality, the definition of the upper Lyapunov exponent, and Theorem 3.9, yield (ii). Statement (iii) is a consequence of (ii) and Theorems 3.11 and 3.7.

Given a function $l: \Omega \rightarrow \mathbb{R}$, we denote $l^{+}(\omega):=\max (l(\omega), 0)$ and $l^{-}(\omega):=$ $\max (-l(\omega), 0)$, so that $l=l^{+}-l^{-}$. The second "majorant" family of cooperative FDEs is

$$
\left\{\begin{array}{l}
\dot{z}_{1}(t)=\widehat{A}_{1}(\omega \cdot t) z_{1}(t)+\widehat{B}_{1}(\omega \cdot t) z_{2}(t)+\widehat{C}_{1}(\omega \cdot t) z_{2}(t-\widehat{\tau}(\widehat{\omega} \cdot t)) \\
\dot{z}_{2}(t)=\widehat{A}_{2}(\omega \cdot t) z_{2}(t)+\widehat{B}_{2}(\omega \cdot t) z_{1}(t)+\widehat{C}_{2}(\omega \cdot t) z_{1}(t-\widehat{\tau}(\widehat{\omega} \cdot t))
\end{array}\right.
$$

for $\widehat{\omega}=\left(\omega,\left[\begin{array}{l}x_{1} \\ x_{2}\end{array}\right]\right) \in \mathcal{A}$, with $A_{i}(\widehat{\omega}) \leq \widehat{A}_{i}(\omega), B_{i}(\widehat{\omega}) \leq\left|B_{i}(\widehat{\omega})\right| \leq \widehat{B}_{i}(\omega)$ and $C_{i}(\widehat{\omega}) \leq$ $\left|C_{i}(\widehat{\omega})\right| \leq \widehat{C}_{i}(\omega)$ for $i=1,2$. In order to ensure these conditions, we take

$$
\begin{aligned}
& \widehat{A}_{1}(\omega):=-a_{1}(\omega)+\kappa_{1 f} b_{1}^{+}(\omega)-\varepsilon_{1 f} b_{1}^{-}(\omega)+\kappa_{2 g} c_{1}^{-}(\omega)-\varepsilon_{2 g} c_{1}^{+}(\omega), \\
& \widehat{B}_{1}(\omega):=\kappa_{3 g}\left|c_{1}(\omega)\right|, \\
& \widehat{C}_{1}(\omega):=\kappa_{4 g}\left|c_{1}(\omega)\right|, \\
& \widehat{A}_{2}(\omega):=-a_{2}(\omega)+\kappa_{1 g} c_{2}^{+}(\omega)-\varepsilon_{1 g} c_{2}^{-}(\omega)+\kappa_{2 f} b_{2}^{-}(\omega)-\varepsilon_{2 f} b_{2}^{+}(\omega), \\
& \widehat{B}_{2}(\omega):=\kappa_{3 f}\left|b_{2}(\omega)\right|, \\
& \widehat{C}_{2}(\omega):=\kappa_{4 f}\left|b_{2}(\omega)\right|,
\end{aligned}
$$


where

$$
\begin{aligned}
& \kappa_{1 f}:=\sup _{\widehat{\omega} \in \mathcal{A}} \dot{f}\left(x_{1}(0)\right), \quad \varepsilon_{1 f}:=\inf _{\widehat{\omega} \in \mathcal{A}} \dot{f}\left(x_{1}(0)\right), \\
& \kappa_{1 g}:=\sup _{\widehat{\omega} \in \mathcal{A}} \dot{g}\left(x_{2}(0)\right), \quad \varepsilon_{1 g}:=\inf _{\widehat{\omega} \in \mathcal{A}} \dot{g}\left(x_{2}(0)\right), \\
& \kappa_{2 f}:=\sup _{\widehat{\omega} \in \mathcal{A}} \dot{f}\left(x_{1}\left(-\tau\left(x_{1}(0), x_{2}(0)\right)\right)\right) \dot{x_{1}}\left(-\tau\left(x_{1}(0), x_{2}(0)\right)\right) \frac{d \tau}{d y_{2}}\left(x_{1}(0), x_{2}(0)\right), \\
& \varepsilon_{2 f}:=\inf _{\widehat{\omega} \in \mathcal{A}} \dot{f}\left(x_{1}\left(-\tau\left(x_{1}(0), x_{2}(0)\right)\right)\right) \dot{x_{1}}\left(-\tau\left(x_{1}(0), x_{2}(0)\right)\right) \frac{d \tau}{d y_{2}}\left(x_{1}(0), x_{2}(0)\right), \\
& \kappa_{2 g}:=\sup _{\widehat{\omega} \in \mathcal{A}} \dot{g}\left(x_{2}\left(-\tau\left(x_{1}(0), x_{2}(0)\right)\right)\right) \dot{x_{2}}\left(-\tau\left(x_{1}(0), x_{2}(0)\right)\right) \frac{d \tau}{d y_{1}}\left(x_{1}(0), x_{2}(0)\right), \\
& \varepsilon_{2 g}:=\inf _{\widehat{\omega} \in \mathcal{A}} \dot{g}\left(x_{2}\left(-\tau\left(x_{1}(0), x_{2}(0)\right)\right)\right) \dot{x_{2}}\left(-\tau\left(x_{1}(0), x_{2}(0)\right)\right) \frac{d \tau}{d y_{1}}\left(x_{1}(0), x_{2}(0)\right), \\
& \kappa_{3 f}:=\sup _{\widehat{\omega} \in \mathcal{A}} \dot{f}\left(x_{1}\left(-\tau\left(x_{1}(0), x_{2}(0)\right)\right)\right)\left|\dot{x_{1}}\left(-\tau\left(x_{1}(0), x_{2}(0)\right)\right) \frac{d \tau}{d y_{1}}\left(x_{1}(0), x_{2}(0)\right)\right|, \\
& \kappa_{3 g}:=\sup _{\widehat{\omega} \in \mathcal{A}} \dot{g}\left(x_{2}\left(-\tau\left(x_{1}(0), x_{2}(0)\right)\right)\right)\left|\dot{x_{2}}\left(-\tau\left(x_{1}(0), x_{2}(0)\right)\right) \frac{d \tau}{d y_{2}}\left(x_{1}(0), x_{2}(0)\right)\right|, \\
& \quad \kappa_{4 f}:=\sup _{\widehat{\omega} \in \mathcal{A}} \dot{f}\left(x_{1}\left(-\tau\left(x_{1}(0), x_{2}(0)\right)\right)\right), \\
& \quad \kappa_{4 g}:=\sup _{\widehat{\omega} \in \mathcal{A}} \dot{g}\left(x_{2}\left(-\tau\left(x_{1}(0), x_{2}(0)\right)\right)\right) .
\end{aligned}
$$

We represent by $\widehat{z}(t, \widehat{\omega}, v)$ the solution of (3.18) with $\widehat{z}(t, \widehat{\omega}, v)(s)=v(s)$ for $s \in$ $[-r, 0]$ for each $\widehat{\omega} \in \mathcal{A}$ and $v \in C_{2}$, denote $\widehat{w}(t, \widehat{\omega}, v)(s)=\widehat{z}(t+s, \widehat{\omega}, v)$ for $s \in[-r, 0]$, and consider the linear skew-product semiflow

$$
\widehat{\zeta}_{L}: \mathbb{R}^{+} \times \mathcal{A} \times C_{2} \rightarrow \mathcal{A} \times C_{2}, \quad(t, \widehat{\omega}, v) \mapsto(\widehat{\omega} \cdot t, \widehat{w}(t, \widehat{\omega}, v)),
$$

which is also monotone (see again Theorem 1.1 of Chapter 5 of [33]). And, for all $\zeta$-minimal set $\mathcal{M} \subset \Omega \times W_{2}^{1, \infty}$ we represent by $\widehat{\lambda}_{\mathcal{M}}$ the upper Lyapunov exponent for $\mathcal{M}$ with respect to the restricted semiflow $\left(\mathcal{M} \times C_{2}, \widehat{\zeta}_{L}, \mathbb{R}^{+}\right)$.

Note that, if we represent (3.17) and (3.18) by $\dot{z}(t)=\bar{L}(\widehat{\omega} \cdot t) z_{t}$ and $\dot{z}(t)=$ $\widehat{L}(\widehat{\omega} \cdot t) z_{t}$, respectively, then

$$
\bar{L}(\widehat{\omega}) z \leq \widehat{L}(\widehat{\omega}) z
$$

for all $\widehat{\omega} \in \mathcal{A}$ and $z \in C_{2}$ with $z \geq 0$.

Theorem 3.13. Suppose that Hypotheses 3.1 hold.

(i) If $\mathcal{M} \subset \Omega \times W_{2}^{1, \infty}$ is a $\zeta$-minimal set, then $\lambda_{\mathcal{M}} \leq \bar{\lambda}_{\mathcal{M}} \leq \hat{\lambda}_{\mathcal{M}}$.

(ii) If $\widehat{\lambda}_{\mathcal{M}}<0$ for all the $\zeta$-minimal sets $\mathcal{M} \subset \Omega \times W_{2}^{1, \infty}$, then all the conclusions of Theorem 3.7 hold.

Proof. Let us fix a $\zeta$-minimal set $\mathcal{M} \subset \Omega \times W_{2}^{1, \infty}$. As said before, the semiflow $\bar{\zeta}_{L}$ is monotone on $\mathcal{M} \times C_{2}$. That is, if $\widehat{\omega} \in \mathcal{M}$ and $v_{1}, v_{2} \in C_{2}$ satisfy $v_{1} \leq v_{2}$, then $\bar{z}\left(t, \widehat{\omega}, v_{1}\right) \leq \bar{z}\left(t, \widehat{\omega}, v_{2}\right)$ for all $t \geq 0$. Consequently, if $\widehat{\omega} \in \mathcal{M}$ and $v \in C_{2}$ then

$$
-\bar{z}\left(t, \widehat{\omega},|v|_{m}\right)=\bar{z}\left(t, \widehat{\omega},-|v|_{m}\right) \leq \bar{z}(t, \widehat{\omega}, v) \leq \bar{z}\left(t, \widehat{\omega},|v|_{m}\right),
$$


for all $t \geq 0$, so that $\|\bar{z}(t, \widehat{\omega}, v)\|_{C_{2}} \leq\left\|\bar{z}\left(t, \widehat{\omega},|v|_{m}\right)\right\|_{C_{2}}$. Therefore,

$$
\begin{aligned}
\bar{\lambda}_{s}^{+}(\widehat{\omega}, v) & :=\limsup _{t \rightarrow \infty} \frac{1}{t} \ln \left\|\bar{z}_{t}(\widehat{\omega}, v)\right\|_{C_{2}} \\
& \leq \limsup _{t \rightarrow \infty} \frac{1}{t} \ln \left\|\bar{z}_{t}\left(\widehat{\omega},|v|_{m}\right)\right\|_{C_{2}}=\bar{\lambda}_{s}^{+}\left(\widehat{\omega},|v|_{m}\right) .
\end{aligned}
$$

On the other hand, (3.19) allows us to apply again Theorem 1.1 of Chapter 5 of [33] in order to obtain $0 \leq \bar{z}\left(t, \widehat{\omega},|v|_{m}\right) \leq \widehat{z}\left(t, \widehat{\omega},|v|_{m}\right)$ for all $t \geq 0$, which ensures that $\left\|\bar{z}\left(t, \widehat{\omega},|v|_{m}\right)\right\|_{C_{2}} \leq\left\|\widehat{z}\left(t, \widehat{\omega},|v|_{m}\right)\right\|_{C_{2}}$. In turn, this inequality yields

$$
\bar{\lambda}_{s}^{+}\left(\widehat{\omega},|v|_{m}\right) \leq \limsup _{t \rightarrow \infty} \frac{1}{t} \ln \left\|\widehat{z}_{t}\left(\widehat{\omega},|v|_{m}\right)\right\|_{C_{2}}=: \widehat{\lambda}_{s}^{+}\left(\widehat{\omega},|v|_{m}\right) .
$$

Altogether, we have $\bar{\lambda}_{s}^{+}(\widehat{\omega}, v) \leq \bar{\lambda}_{s}^{+}\left(\widehat{\omega},|v|_{m}\right) \leq \widehat{\lambda}_{s}^{+}\left(\widehat{\omega},|v|_{m}\right) \leq \widehat{\lambda}_{\mathcal{M}}$ for all $\widehat{\omega} \in \mathcal{M}$ and $v \in C_{2}$. Consequently, $\bar{\lambda}_{\mathcal{M}} \leq \hat{\lambda}_{\mathcal{M}}$. Applying Theorem 3.12(ii), we have $\lambda_{\mathcal{M}} \leq \bar{\lambda}_{\mathcal{M}} \leq \hat{\lambda}_{\mathcal{M}}$. This proves (i). Statement (ii) is an immediate consequence of (i) and Theorems 3.11 and 3.7.

Remark 3.14. 1. Regarding the upper Lyapunov exponent of a minimal subset of $\Omega \times W_{2}^{1, \infty}$, one of the advantages of working with cooperative families (as is the case of (3.17) and (3.18)) is that the exponent can be obtained by computing (2.4) for any solution of a particular one of the systems corresponding to a strongly positive initial state; that is, by a vector $v \in C_{2}$ such that $v_{i}(s)>0$ for all $s \in[-r, 0]$ and $i=1,2$. To check this assertion, we use the notation corresponding to the flow $\bar{\zeta}_{L}$, and note that: (1) $\bar{\lambda}_{s}(\widehat{\omega}, v) \leq \bar{\lambda}_{s}\left(\widehat{\omega},|v|_{m}\right)$ (see the proof of Theorem 3.13(i)); (2) $|v|_{m}=v$ if $v$ is positive; and (3) for all pair of strongly positive vectors $v$ and $w$ in $C_{2}$, there exists a constant $\alpha>0$ such that $(1 / \alpha) w \leq v \leq \alpha w$. And we also use the ergodic uniqueness of the base flow, to ensure the independence with respect to the chosen system.

2. Note also that, in the case of (3.18), the coefficients $\widehat{A}_{i}, \widehat{B}_{i}$, and $\widehat{C}_{i}$ are evaluated along the semiorbits of the base flow $(\Omega, \sigma, \mathbb{R})$ instead of those of the flow over $\mathcal{A}$ (which are, in general, unknown). Although the equations still depend on the semiorbits on $\mathcal{A}$ through the delay, there are many results on exponential stability which are given in terms of $\widehat{A}_{i}, \widehat{B}_{i}$, and $\widehat{C}_{i}$ and independent of the delay. Basically, they consist in comparing the negativeness of $\widehat{A}_{i}$ with the positiveness of $\widehat{B}_{i}$ and $\widehat{C}_{i}$ : see e.g. [10], [34], [38], [2], and references therein. An example of this situation is given in the proof of Proposition 4.3.

\section{Numerical EXPERIMENTS}

In this section we perform numerical experiments on the family of SDDEs

$$
\left\{\begin{aligned}
\dot{y}_{1}(t)= & \left(-1+0.3 \cos \left(\theta_{1}+\sqrt{2} t\right)\right) y_{1}(t) \\
& +\left(-1.5+\sin \left(\theta_{2}+t\right)\right) \tanh \left(y_{1}(t)\right) \\
& +\left(1.4-\sin \left(\theta_{2}+t\right)\right) \tanh \left(y_{2}\left(t-\tau\left(y_{1}, y_{2}\right)\right)\right)+0.4 \\
\dot{y}_{2}(t)= & \left(-1.2+0.3 \sin \left(\theta_{1}+\sqrt{2} t\right)\right) y_{2}(t) \\
& +\left(1.3-0.3 \cos \left(\theta_{2}+t\right)\right) \tanh \left(y_{1}\left(t-\tau\left(y_{1}, y_{2}\right)\right)\right) \\
& +\left(-1+0.3 \cos \left(\theta_{2}+t\right)\right) \tanh \left(y_{2}(t)\right)+0.4
\end{aligned}\right.
$$


for $t \geq 0$, where $\left(\theta_{1}, \theta_{2}\right) \in \mathbb{T}^{2}$ : for this example $\Omega$ is the 2-torus, which we identify with $(\mathbb{R} /[0,2 \pi])^{2}$, and the (Kronecker) flow $\sigma$ is given by $\sigma\left(t,\left(\theta_{1}, \theta_{2}\right)\right) \equiv\left(\theta_{1}, \theta_{2}\right) \cdot t:=$ $\left(\theta_{1}+\sqrt{2} t, \theta_{2}+t\right) \in \mathbb{T}^{2}$. The family (4.1) is obtained via the hull procedure from the single $\operatorname{SDDE}(4.1)_{(0,0)}$. Therefore, it satisfies Hypotheses 3.1 whenever the delay $\tau: \mathbb{R}^{2} \rightarrow[0, r]$ is $C^{1}$, and the results of Theorem 3.2 apply: the skew product semiflow $\left(\mathbb{T}^{2} \times W_{2}^{1, \infty}, \zeta, \mathbb{R}^{+}\right)$induced by the solutions of (4.1) has a connected global attractor $\mathcal{A}$.

Our first purpose is to estimate a subset of $\mathbb{T}^{2} \times W_{2}^{1, \infty}$ as small as possible in which the attractor $\mathcal{A}$ lies. This procedure, completed in Corollary 4.2, will not depend on the specific expression of the delay. But later we will take

$$
\tau^{\delta}\left(y_{1}, y_{2}\right):=1-\sin \left(\delta\left(y_{1}+y_{2}\right)\right)
$$

in order to prove, in Proposition 4.3, that the attractor is a copy of the base.

Given $x_{0} \in W_{2}^{1, \infty}$, we represent by $y\left(t, \theta_{1}, \theta_{2}, x_{0}\right)$ the solution of $(4.1)_{\left(\theta_{1}, \theta_{2}\right)}$ with $y\left(s, \theta_{1}, \theta_{2}, x_{0}\right)=x_{0}(s)$ for $s \in[-r, 0]$.

4.1. A set containing the global attractor. Let us begin with the estimation of a "small" subset of $\mathbb{T}^{2} \times W_{2}^{1, \infty}$ containing the attractor $\mathcal{A}$. To this end we define the functions $F_{i}: \mathbb{T}^{2} \times \mathbb{R}^{2} \rightarrow \mathbb{R}^{2}$ for $i=1,2$ by

$$
\begin{aligned}
F_{1}\left(\theta_{1}, \theta_{2}, k_{1}, k_{2}\right):= & \left(-1+0.3 \cos \left(\theta_{1}\right)\right) k_{1}+\left(-1.5+\sin \left(\theta_{2}\right)\right) \tanh \left(k_{1}\right) \\
& +\left(1.4-\sin \left(\theta_{2}\right)\right) \tanh \left(k_{2}\right)+0.4 \\
F_{2}\left(\theta_{1}, \theta_{2}, k_{1}, k_{2}\right):= & \left(-1.2+0.3 \sin \left(\theta_{1}\right)\right) k_{2}+\left(1.3-0.3 \cos \left(\theta_{2}\right)\right) \tanh \left(k_{1}\right) \\
& +\left(-1+0.3 \cos \left(\theta_{2}\right)\right) \tanh \left(k_{2}\right)+0.4
\end{aligned}
$$

so that $(4.1)_{\left(\theta_{1}, \theta_{2}\right)}$ agrees with

$$
\left\{\begin{array}{l}
\dot{y}_{1}(t)=F_{1}\left(\left(\theta_{1}, \theta_{2}\right) \cdot t, y_{1}(t), y_{2}\left(t-\tau\left(y_{1}, y_{2}\right)\right)\right), \\
\dot{y}_{2}(t)=F_{2}\left(\left(\theta_{1}, \theta_{2}\right) \cdot t, y_{1}\left(t-\tau\left(y_{1}, y_{2}\right)\right), y_{2}(t)\right)
\end{array}\right.
$$

for $t \geq 0$. Below we will find sequences $\left(k_{1 n}^{ \pm}\right)$and $\left(k_{2 n}^{ \pm}\right)$such that

$$
k_{i n}^{-}<k_{i, n+1}^{-}<k_{i, n+1}^{+}<k_{i, n}^{+} \text {for } i<1,2 \text { and } n \geq 0 \text {, }
$$

and such that if $\mathcal{R}_{n}:=\left[k_{1, n}^{-}, k_{1, n}^{+}\right] \times\left[k_{2, n}^{-}, k_{2, n}^{+}\right] \subset \mathbb{R}^{2}$ for $n \geq 0$, then

Proposition 4.1. $\mathcal{A} \subseteq \mathbb{T}^{2} \times \widetilde{\mathcal{R}}_{n}$ for $\widetilde{\mathcal{R}}_{n}:=\left\{\varphi \in W_{2}^{1, \infty} \mid \varphi:[-r, 0] \rightarrow \mathcal{R}_{n}\right\}$.

For ease of notation we drop the explicit dependence of $F_{i}$ on $\left(\theta_{1}, \theta_{2}\right)$. We build the four sequences $\left(k_{1 n}^{ \pm}\right)_{n \geq 1}$ and $\left(k_{2 n}^{ \pm}\right)_{n \geq 0}$ following the next steps:

$\mathbf{S}_{0}$ Initialize the sequences $\left(k_{1 n}^{ \pm}\right)_{n \geq 0}$ and $\left(k_{2 n}^{ \pm}\right)_{n \geq 0}$ to the following values:

$$
k_{i 0}^{+}:=+\infty \quad \text { and } \quad k_{i 0}^{-}:=-\infty \quad \text { for } i=1,2 .
$$

$\mathbf{S}_{11}^{+}$We set $F_{11}^{+}(k):=F_{1}\left(k, k_{20}^{+}\right)$and note that $F_{1}\left(k, k_{2}\right) \leq F_{11}^{+}(k)$ for all $k_{2} \leq k_{20}^{+}$ and all $\left(\theta_{1}, \theta_{2}\right) \in \mathbb{T}^{2}$. We want to find a $k_{11}^{+}$such that $F_{11}^{+}(k)<0$ for all $\left(\theta_{1}, \theta_{2}\right) \in \mathbb{T}^{2}$ whenever $k>k_{11}^{+}$. Note that $F_{11}^{+}(0)=\left(1.4-\sin \left(\theta_{2}\right)\right)+0.4>0$ and hence $k_{11}^{+}$must necessarily be greater than 0 . For $k>0$ and all $\left(\theta_{1}, \theta_{2}\right) \in \mathbb{T}^{2}$, $F_{11}^{+}(k) \leq G_{11}^{+}(k)$, where

$$
\begin{array}{r}
G_{11}^{+}(k):=-0.7 k-1.5 \tanh (k)+1.4 \tanh \left(k_{20}^{+}\right) \\
+\left|\tanh (k)-\tanh \left(k_{20}^{+}\right)\right|+0.4 .
\end{array}
$$


This is a strictly decreasing function, so that we achieve our goal by taking $k_{11}^{+}$as the unique point with $G_{11}^{+}\left(k_{11}^{+}\right)=0$.

$\mathbf{S}_{11}^{-}$We set $F_{11}^{-}(k):=F_{1}\left(k, k_{20}^{-}\right)$and observe that $F_{1}\left(k, k_{2}\right)>F_{11}^{-}(k)$ for all $k_{2} \geq$ $k_{20}^{-}$and all $\left(\theta_{1}, \theta_{2}\right) \in \mathbb{T}^{2}$. We want to find a $k_{11}^{-}$such that $F_{11}^{-}(k)>0$ for all $\left(\theta_{1}, \theta_{2}\right) \in \mathbb{T}^{2}$ whenever $k<k_{11}^{-}$. Let us define the continuous and strictly decreasing function

$$
G_{11}^{-}(k):=\left\{\begin{array}{cc}
\left.-0.7 k-1.5 \tanh (k)+1.4 \tanh \left(k_{20}^{-}\right)\right) & \\
-\left|\tanh (k)-\tanh \left(k_{20}^{-}\right)\right|+0.4 & \text { if } k<0, \\
\left.-1.3 k-1.5 \tanh (k)+1.4 \tanh \left(k_{20}^{-}\right)\right) & \text {if } k \geq 0 . \\
-\left|\tanh (k)-\tanh \left(k_{20}^{-}\right)\right|+0.4 & \text {. }
\end{array}\right.
$$

Then $F_{11}^{-}(k) \geq G_{11}^{-}(k)$ for all $k \in \mathbb{R}$ and all $\left(\theta_{1}, \theta_{2}\right) \in \mathbb{T}^{2}$. We take $k_{11}^{-}$such that $G_{11}^{-}\left(k_{11}^{-}\right)=0$.

$\mathbf{S}_{21}^{+}$We set $F_{21}^{+}(k):=F_{2}\left(k_{10}^{+}, k\right)$ and note that $F_{2}\left(k_{1}, k\right) \leq F_{21}^{+}(k)$ for all $k_{1} \leq k_{10}^{+}$ and all $\left(\theta_{1}, \theta_{2}\right) \in \mathbb{T}^{2}$. As in $\mathbf{S}_{11}^{+}$, we look for $k_{21}^{+}$(which must be greater than 0 ) such that $F_{21}^{+}(k)<0$ for all $\left(\theta_{1}, \theta_{2}\right) \in \mathbb{T}^{2}$ whenever $k>k_{21}^{+}$, what we achieve by taking $k_{21}^{+}$as the unique zero of the strictly decreasing function

$$
\begin{aligned}
G_{21}^{+}(k):=-0.9 k & -\tanh (k)+1.3 \tanh \left(k_{10}^{+}\right) \\
& +0.3\left|\tanh (k)-\tanh \left(k_{10}^{+}\right)\right|+0.4 .
\end{aligned}
$$

$\mathbf{S}_{21}^{-}$We set $F_{21}^{-}(k):=F_{2}\left(k_{10}^{-}, k\right)$ and note that $F_{2}\left(k, k_{2}\right) \geq F_{21}^{-}(k)$ for all $k \geq k_{10}^{-}$ and all $\left(\theta_{1}, \theta_{2}\right) \in \mathbb{T}^{2}$. Reasoning as in $\mathbf{S} 11^{-}$we define $k_{21}^{-}$as the unique zero of the continuous and strictly decreasing function

$$
G_{21}^{-}:=\left\{\begin{array}{cc}
-0.9 k-\tanh (k)+1.3 \tanh \left(k_{10}^{-}\right) & \text {if } k<0, \\
-0.3\left|\tanh (k)-\tanh \left(k_{10}^{-}\right)\right|+0.4 & \text { if } k \geq 0 . \\
-1.5 k-\tanh (k)+1.3 \tanh \left(k_{10}^{-}\right) & \text {. }
\end{array}\right.
$$

In the steps $\mathbf{S}_{1 n}^{ \pm}$we define $k_{1 n}^{ \pm}$by repeating steps $\mathbf{S}_{11}^{ \pm}$with $k_{2, n-1}^{ \pm}$in place of $k_{20}^{ \pm}$; and in the steps $\mathbf{S}_{2 n}^{ \pm}$we define $k_{2 n}^{ \pm}$by repeating steps $\mathbf{S}_{21}^{ \pm}$with $k_{1, n-1}^{ \pm}$in place of $k_{10}^{ \pm}$. It is not hard (by applying an induction procedure based on comparing the functions $G_{i n}^{ \pm}$, whose zeroes determine the four sequences) that (4.2) hold.

Proof of Proposition 4.1. Is is obvious that $\mathcal{A} \subset \widetilde{\mathcal{R}}_{0}=\mathbb{R}^{2}$. We will assume that $\mathcal{A} \subset \widetilde{\mathcal{R}}_{n-1}$ and prove that $\mathcal{A} \subset \widetilde{\mathcal{R}}_{n}$. Recall that $\mathcal{A}$ is composed by the globally defined and bounded orbits: see Remarks 2.2.3 and 2.7.1. Let us assume that there exists $\left(\bar{\theta}_{1}, \bar{\theta}_{2}, \bar{x}\right) \in \mathcal{A}$ such that $\bar{x} \notin \widetilde{\mathcal{R}}_{n}$. There is no restriction in assuming that $\bar{x}(0) \notin \mathcal{R}_{n}$. We will get a contradiction supposing that $\bar{k}_{1}=\bar{x}_{1}(0)>k_{1 n}^{+}$, since the remaining possibilities are analyzed in an analogous way. We define $\mathcal{K}_{2}:=$ $\left\{x_{2}(s) \mid\left(\theta_{1}, \theta_{2}, x\right) \in \mathcal{A}, s \in[-r, 0]\right\}$, which is a compact subset of $\left[k_{2, n-1}^{-}, k_{2, n-1}^{+}\right]$, and $\delta:=\max _{\left(\theta_{1}, \theta_{2}\right) \in \mathbb{T}^{2}, k_{2} \in \mathcal{K}_{2}} F_{1}\left(\theta_{1}, \theta_{2}, k_{1 n}^{+}, k_{2}\right)$. Observe that the expression of $F_{1}$ and the inequalities in $\mathbf{S}_{1 n}^{+}$ensure that $F_{1}\left(\theta_{1}, \theta_{2}, k_{1}, k_{2}\right)<F_{1}\left(\theta_{1}, \theta_{2}, k_{1 n}^{+}, k_{2}\right) \leq \delta<0$ whenever $k_{1}>k_{1 n}^{+}, k_{2} \in \mathcal{K}_{2}$ and $\left(\theta_{1}, \theta_{2}\right) \in \mathbb{T}^{2}$.

Let us represent by $\left\{\left(\left(\bar{\theta}_{1}, \bar{\theta}_{2}\right) \cdot t, \bar{u}(t)\right) \mid t \in \mathbb{R}\right\} \subset \mathcal{A}$ a complete orbit for $\left(\bar{\theta}_{1}, \bar{\theta}_{2}, \bar{x}\right)$ (which is its value at 0 ), so that: $\bar{y}(t):=\bar{u}(t)(0)$ satisfies $(4.1)_{\left(\bar{\theta}_{1}, \bar{\theta}_{2}\right)}$ for all $t \in \mathbb{R}$, and the second component $\bar{y}_{2}$ takes values in $\mathcal{K}_{2}$. Then $\dot{\bar{y}}_{1}(0) \leq \delta$, and it is easy 
to deduce by contradiction that $\dot{\bar{y}}_{1}(t) \leq \delta$ for all $t<0$. But this precludes the boundedness of $\bar{y}(t)$, which is the sought-for contradiction.

The fact that Proposition 4.1 is valid for $n \geq 0$ proves the next assertion.

Corollary 4.2. Let us define $\widetilde{\mathcal{R}}:=\bigcap_{n \geq 0} \mathcal{R}_{n}$. Then $\mathcal{A} \subseteq \mathbb{T}^{2} \times \widetilde{\mathcal{R}}$.

Note also that, if $k_{i}^{ \pm}:=\lim _{n \rightarrow \infty} k_{i n}^{ \pm}$for $i=1,2$ and $\mathcal{R}:=\left[k_{1}^{-}, k_{1}^{+}\right] \times\left[k_{2}^{-}, k_{2}^{+}\right] \subset \mathbb{R}^{2}$ then $\widetilde{\mathcal{R}}=\left\{\varphi \in W_{2}^{1, \infty} \mid \varphi:[-r, 0] \rightarrow \mathcal{R}\right\}$. We also remark that, for $i=1,2$, the functions $G_{i n}^{ \pm}$do not depend on the delay, and hence the area containing the global attractor is independent of the particular choice of $\tau$ : for all the families (4.1) we obtain as estimate of the rectangle $\mathcal{R}=[0.2910,0.5705] \times[0.3090,0.5999]$. The sequences of zeros are obtained using a standard Matlab command, working with a tolerance of $10^{-5}$. We will make use of the less accurate bounds

$$
\mathcal{R} \subset[0.28,0.58] \times[0.30,0.61]
$$

in order to prove the next result:

Proposition 4.3. Let us take the delay $\tau^{\delta}\left(y_{1}, y_{2}\right):=1-\sin \left(\delta\left(y_{1}+y_{2}\right)\right)$. Then, if $|\delta|$ is small enough, the upper Lyapunov exponent of the corresponding global attractor $\mathcal{A}^{\delta}$ is strictly negative, and consequently $\mathcal{A}^{\delta}$ is a copy of $\mathbb{T}^{2}$.

Proof. Note that the second assertion follows from the first one and Theorem 3.7. Let us consider the family $(3.18)_{\delta}$ constructed from (4.1) for the delay $\tau_{\delta}$, given by

$$
\begin{aligned}
& \widehat{A}_{1}^{\delta}\left(\theta_{1}, \theta_{2}\right):=-1+0.3 \cos \theta_{1}-\varepsilon_{1 f}^{\delta}\left(1.5-\sin \theta_{2}\right)-\varepsilon_{2 g}^{\delta}\left(1.4-\sin \theta_{2}\right), \\
& \widehat{B}_{1}^{\delta}\left(\theta_{1}, \theta_{2}\right):=\kappa_{3 g}^{\delta}\left(1.4-\sin \theta_{2}\right), \\
& \widehat{C}_{1}^{\delta}\left(\theta_{1}, \theta_{2}\right):=\kappa_{4 g}^{\delta}\left(1.4-\sin \theta_{2}\right), \\
& \widehat{A}_{2}^{\delta}\left(\theta_{1}, \theta_{2}\right):=-1.2+0.3 \sin \theta_{1}-\varepsilon_{1 g}^{\delta}\left(1-0.3 \cos \theta_{2}\right)-\varepsilon_{2 f}^{\delta}\left(1.3-0.3 \cos \theta_{2}\right), \\
& \widehat{B}_{2}^{\delta}\left(\theta_{1}, \theta_{2}\right):=\kappa_{3 f}^{\delta}\left(1.3-0.3 \cos \theta_{2}\right), \\
& \widehat{C}_{2}^{\delta}\left(\theta_{1}, \theta_{2}\right):=\kappa_{4 f}^{\delta}\left(1.3-0.3 \cos \theta_{2}\right),
\end{aligned}
$$

where the constants are defined by the expressions given before Theorem 3.13, now corresponding to $\tau^{\delta}$ and $\mathcal{A}^{\delta}$. According to Proposition 5.3 of of [27], it is enough to prove that

$$
\widehat{A}_{1}^{\delta}+\widehat{B}_{1}^{\delta}+\widehat{C}_{1}^{\delta}<0 \quad \text { and } \quad \widehat{A}_{2}^{\delta}+\widehat{B}_{2}^{\delta}+\widehat{C}_{2}^{\delta}<0 .
$$

Note that $f$ and $g$ are given in our case by $\tan h$, whose derivative decreases in $\mathbb{R}^{+}$, and that Corollary 4.2 and $(4.3)$ yield $x(s) \in[0.28,0.58] \times[0.30,0.61]$ for all $s \in[-2,0]$ and $\left(\theta_{1}, \theta_{2}, x\right) \in \mathcal{A}^{\delta}$. This ensures that $\varepsilon_{1 f}^{\delta} \geq \dot{f}(0.58), \varepsilon_{1 g}^{\delta} \geq \dot{g}(0.61)$, $\kappa_{4 g}^{\delta} \leq \dot{g}(0.30)$ and $\kappa_{4 f}^{\delta} \leq \dot{f}(0.28)$. Therefore,

$$
\begin{aligned}
\widehat{A}_{1}^{\delta}+\widehat{B}_{1}^{\delta}+\widehat{C}_{1}^{\delta} & \leq-0.7-2.5 \dot{f}(0.58)+2.4 \dot{g}(0.30)+2.4\left(\left|\kappa_{3 g}^{\delta}\right|+\left|\varepsilon_{2 g}^{\delta}\right|\right) \\
& <-0.3+2.4\left(\left|\kappa_{3 g}^{\delta}\right|+\left|\varepsilon_{2 g}^{\delta}\right|\right) \\
\widehat{A}_{2}^{\delta}+\widehat{B}_{2}^{\delta}+\widehat{C}_{2}^{\delta} & \leq-0.9-1.3 \dot{g}(0.61)+1.6 \dot{f}(0.28)+1.6\left(\left|\kappa_{3 f}^{\delta}\right|+\left|\varepsilon_{2 f}^{\delta}\right|\right) \\
& <-0.3+1.6\left(\left|\kappa_{3 f}^{\delta}\right|+\left|\varepsilon_{2 f}^{\delta}\right|\right) .
\end{aligned}
$$

It is also easy to deduce from the compactness of $\mathcal{A}$ and from the expression of $\tau^{\delta}$ that there exists $c>0$ such that $\left|\kappa_{3 g}^{\delta}\right|,\left|\varepsilon_{2 g}^{\delta}\right|,\left|\kappa_{3 f}^{\delta}\right|$ and $\left|\varepsilon_{2 f}^{\delta}\right|$ are bounded by $c|\delta|$, which shows that (4.4) holds if $|\delta|$ is small enough. 
4.2. Numerical simulations. In what follows we report on numerical simulations of (4.1) with delay $\tau\left(y_{1}, y_{2}\right):=1-\sin \left(0.1\left(y_{1}+y_{2}\right)\right)$. All our computations are done with the Matlab function ddesd. When not specified, we use the default options. We simulate the dynamic, visualize a set $\mathcal{A}$ that attracts all numerical solutions and give numerical evidence that $\mathcal{A}$ is a one copy of the base (so that $\delta=0.1$ is one of the values provided by Proposition 4.3).

For convenience, we choose a finite set $X \subset W_{2}^{1, \infty}$ of initial conditions with constant value in $[-2,0]$ and we solve $(4.1)$ for each $x_{0} \in X$ and for a $\left(\theta_{1}^{0}, \theta_{2}^{0}\right)$ fixed: $(0,0)$ for Figure 1 . Let us call $y\left(t, x_{0}\right):=y\left(t, 0,0, x_{0}\right)$. In Figure 1 on the left we plot in the $y_{1}-y_{2}$ plane the solutions $y\left(t, x_{0}\right)$ of $(4.1)_{(0,0)}$ for $x_{0} \in X$ computed up to time $T=2000$. On the right, for the same set $X$, we plot $\left(y_{1}\left(t, x_{0}\right), y_{2}\left(t, x_{0}\right), \theta_{2}(t)\right)$ for $t \in[1000,2000]$, after getting rid of transient.

Figure 1. Simulations of $(4.1)_{(0,0)}$. Left: different solutions converging to $\mathcal{A}$. Right: plot of $\left(y_{1}(t), y_{2}(t), \theta_{2}(t)\right)$ for $t \in[1000,2000]$ for the same set of solutions.
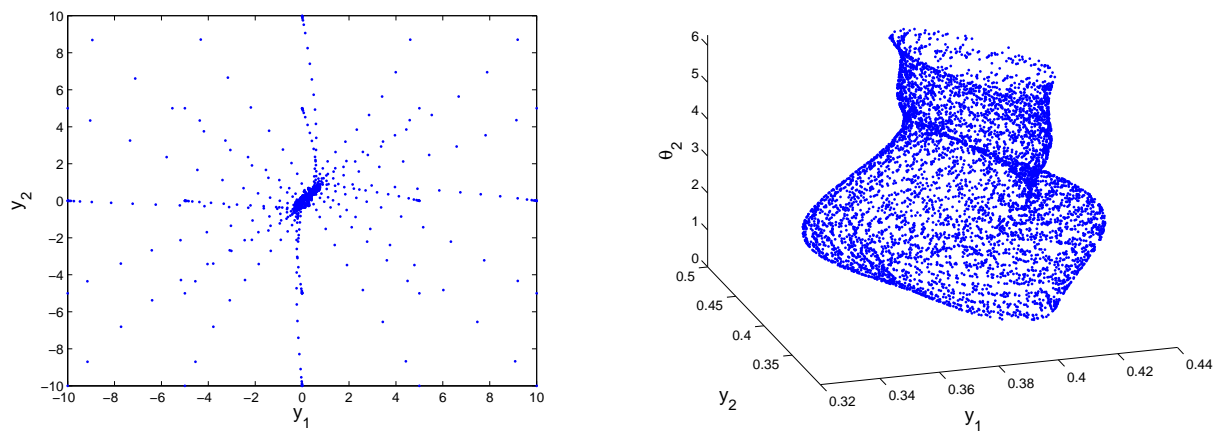

In Figure 2, we depict the numerical attractor for $(4.1)_{\left(\theta_{1}, \theta_{2}\right)}$ with $\left(\theta_{1}, \theta_{2}\right) \neq(0,0)$ and compare it with that obtained for $(0,0)$. On the left we plot the attractor in the $y_{1}-y_{2}$ plane for $(0,0)$. On the right, in blue we plot the numerical solutions for $(0,0)$ and in red the numerical solutions for $(\pi / 3, \pi / 2)$. Similar plots are obtained for other systems in the family. Note that the numerical attractors are contained in the set $\mathcal{R}$ obtained in Section 4.1. All the above computations are performed with RelTol $=10^{-9}$ in ddesd. We point out that the plots in Figure 2 do not change if, instead of considering the whole set of initial conditions $x_{0} \in X$, we consider only one initial condition $x_{0}$. This fact seems to indicate that the attractor has negative Lyapunov exponent and hence (see Theorem 3.7) is a copy of the base, so that if we fix $\left(\theta_{1}, \theta_{2}\right)$ in (4.1), all solutions corresponding to different initial conditions will eventually converge. This fact is also confirmed by Figure 3 , where we plot $y_{1}\left(t, x_{0}\right)$ and $y_{2}\left(t, x_{0}\right)$ in the time interval $[2,40]$ for all $x_{0} \in X$.

As a matter of fact, Proposition 4.3 guarantees that, if $\delta=0.1$ "is small enough", then the global attractor $\mathcal{A}$ is indeed a copy of the base; that is, the graph of a continuous function $a: \mathbb{T}^{2} \rightarrow \widetilde{\mathcal{R}}$ with $\widetilde{\mathcal{R}}$ defined in Corollary 4.2. But it is not clear how to determine if $\delta=0.1$ is in that case. However, we obtain two numerical evidences, which we describe to complete the paper. 
Figure 2. Plot of $\left(y_{1}(t), y_{2}(t)\right)$ for different numerical solutions. Left: $\left(\theta_{1}^{0}, \theta_{2}^{0}\right)=(0,0)$. Right: in blue $\left(\theta_{1}^{0}, \theta_{2}^{0}\right)=(0,0)$, in red $\left(\theta_{1}^{0}, \theta_{2}^{0}\right)=(\pi / 3, \pi / 2)$.
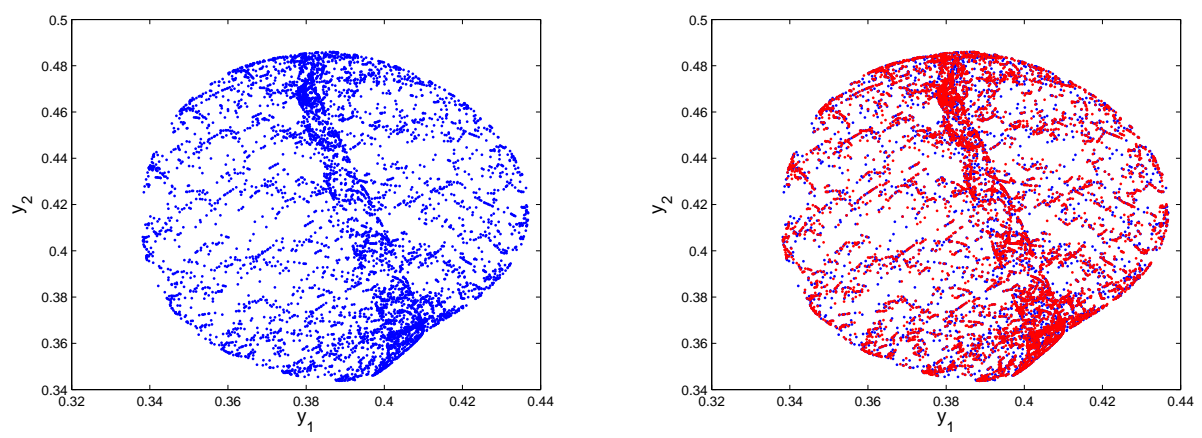

Figure 3. Plot of $\left(t, y_{1}\left(t, x_{0}\right)\right)$ and $\left(t, y_{2}\left(t, x_{0}\right)\right)$ for different numerical solutions.
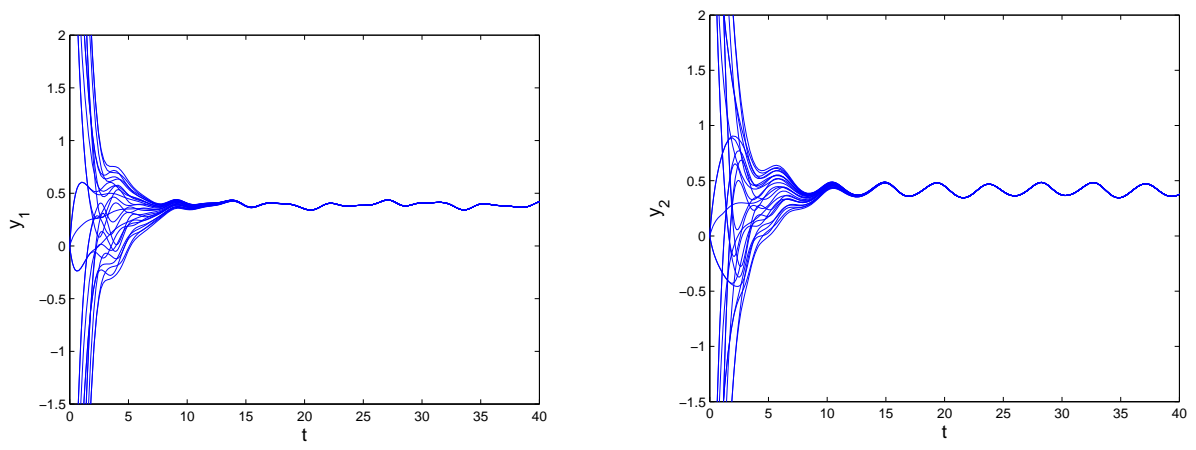

Assume that $\mathcal{A}$ is the graph of $a$. Then $a(\omega)(0)=\lim _{t \rightarrow \infty} y\left(t, \omega(-t), x_{0}\right)$ for any $x_{0}$, as deduced for instance from Theorem 3.5. And, in addition, the components of the map $\mathbb{T}^{2} \rightarrow \mathbb{R}^{2},\left(\theta_{1}, \theta_{2}\right) \mapsto a\left(\theta_{1}, \theta_{2}\right)(0)$ define two copies of $\mathbb{T}^{2}$ in $\mathbb{T}^{2} \times \mathbb{R}$. So that we first define a uniform grid of base points $\left(\theta_{1}^{j}, \theta_{2}^{k}\right), j, k=1, \ldots, 20$. We fix a tolerance tol $=10^{-6}$ and an $x_{0} \in X$ and compute $y^{j k}:=y\left(T,\left(\theta_{1}^{j}, \theta_{2}^{k}\right) \cdot(-T), x_{0}\right)$ for $j, k=1, \ldots, 20$, where $T$ is such that the distance between the $y^{j k}$ 's computed at time $T$ and time $(T-5)$ is less than the fixed tolerance. In Figure 4 on the left we plot $\left(\theta_{1}^{j}, \theta_{2}^{k}, y_{1}^{j k}\right)$ and on the right we plot $\left(\theta_{1}^{j}, \theta_{2}^{k}, y_{2}^{j k}\right)$.

We obtain the same plots for any other initial condition $x_{0}$ we consider. Observe that the sections corresponding to $\left(0, \theta^{k}\right)$ and $\left(2 \pi, \theta^{k}\right)$ agree, likewise those for $\left(\theta^{j}, 0\right)$ and $\left(\theta^{j}, 2 \pi\right)$. That is, both plots seem to be graphs of continuous functions $\mathbb{T}^{2} \rightarrow \mathbb{R}$, as expected. This is the first numerical evidence of the fact that we have indeed a copy of the base. Note also that we are acting in a "pullback" sense in order to depict the attractor.

For the second evidence we will combine forward and backward methods. First, we obtain three sections of the plots in Figure 4. For that, we take a denser grid of 
Figure 4. Mesh of $\left(\theta_{1}^{j}, \theta_{2}^{k}, y_{1}^{j k}\right)$ on the left and $\left(\theta_{1}^{j}, \theta_{2}^{k}, y_{2}^{j k}\right)$ on the right for any initial condition and $T=30$.
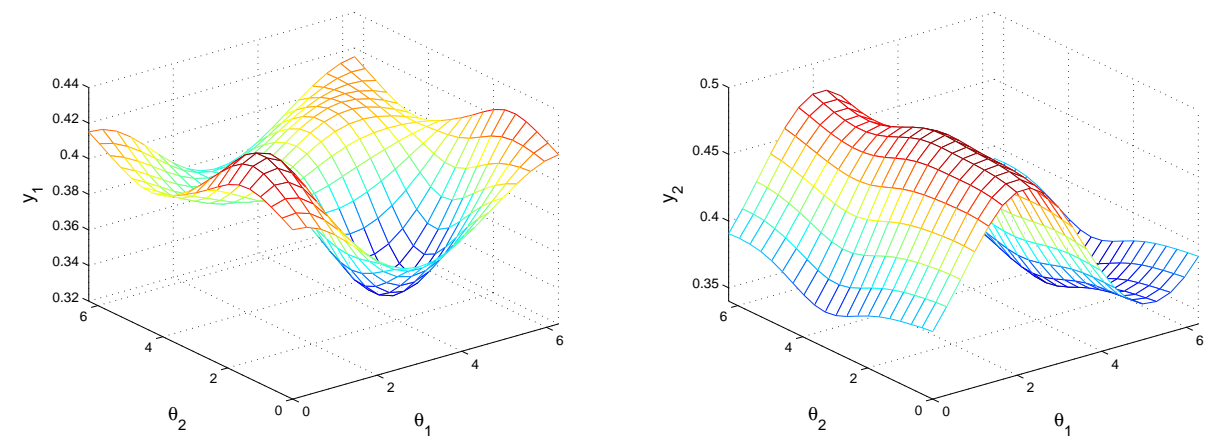

50 points for $\theta_{1}^{j}$, and obtain $y^{j}:=u\left(T,\left(\theta_{1}^{j}, \theta_{2}\right) \cdot T, x_{0}\right)(0)$ for $T=30$ and three values of $\theta_{2}: 0, \pi / 3, \pi / 2$. In Figure 5 we plot with continuous line the three graphics for $\left(\theta_{1}^{j}, y_{1}^{j}\right)$ on the left and for $\left(\theta_{1}^{j}, y_{2}^{j}\right)$ on the right. Let now act in a "forward sense": we consider three Poincaré sections for a fixed $\theta_{2}$, again for $0, \pi / 3, \pi / 2$. The markers in Figure 5 correspond to the sets of points $\left(\theta_{1}(t), y_{1}(t)\right)$ (left) and $\left(\theta_{1}(t), y_{2}(t)\right)$ (right) on the three sections after getting rid of transient. And this time we take a different type of initial condition: $x_{0}(s):=(-t, \cos (s))$ for $s \in[-2,0]$. As expected, we obtained the same plot for any other initial condition we considered. The fact that the curves in Figure 5 overlap constitutes the second evidence we referred to.

Figure 5. Sections of the copies of the base of Figure 4 for $\theta_{2}=$ $0, \pi / 3, \pi / 2$ (continuous lines). Plots of $\left(\theta_{1}(t), y_{1}(t)\right),\left(\theta_{1}(t), y_{2}(t)\right)$ on the Poincaré sections $\theta_{2}=0, \pi / 3, \pi / 2$ for $T \in[1000,2000]$, with initial condition $x_{0}(s):=(-s, \cos (s))$ (markers).
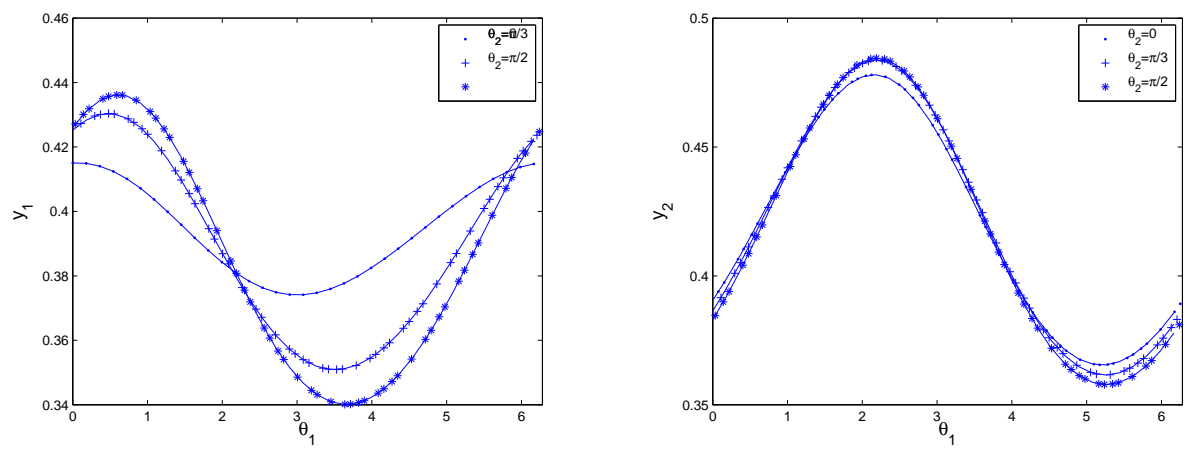

\section{REFERENCES}

[1] L. Arnold, Random Dynamical Systems, Springer-Verlag, Berlin, Heidelberg, New York, 1998. 
[2] O. Arratia, R. Obaya, M.E. Sansaturio, Applications of the exponential ordering in the study of almost-periodic delayed Hopfield neural networks, Physica D 241 (2012), 1551-1566

[3] T. Caraballo, X. Han, Applied Nonautonomous and Random Dynamical Systems, SpringerBriefs in Mathematics, 2016.

[4] T. Caraballo, J. Langa, R. Obaya, Pullback, forwards and chaotic dynamics in 1-D nonautonomous linear-dissipative equations, Nonlinearity 30 (1) (2017), 274-299.

[5] A. Carvalho, J. Langa, J. Robinson, Attractors for Infinite-dimensional Non-autonomous Dynamical Systems, Appl. Math. Sci. 182, Springer, 2013.

[6] D. Cheban, P. Kloeden, B. Schmalfuss; Pullback attractors in dissipative nonautonomous differential equations under discretization, J. Dynam. Differ. Eqs. 13 (1) 2001, 185-213.

[7] S.-N. Chow, H. Leiva, Dynamical spectrum for time dependent linear systems in Banach spaces, Japan J. Indust. Appl. Math. 11 (1994), 379-415.

[8] S.-N. Chow, H. Leiva, Existence and roughness of the exponential dichotomy for skewproduct semiflow in Banach spaces, J. Differential Equations 120 (1995), 429-477.

[9] A.M. Fink, Almost Periodic Differential Equations, Lecture Notes in Math. 377, SpringerVerlag, Berlin, Heidelberg, New York, 1974.

[10] J.K. Hale, Theory of Functional Differential Equations, Springer-Verlag, Berlin, Heidelberg, New York, 1977.

[11] J.K. Hale, Asymptotic Behavior of Dissipative Systems, Math. Surveys Monogr. 25, Amer. Math. Soc., Providence, 1988.

[12] F. Hartung, On differentiability of solutions with respect to parameters in a class of functional differential equations, Funct. Differ. Equ. 4 (1)-(2) (1997), 65-79.

[13] F. Hartung, Differentiability of solutions with respect to the initial data in differential equations with state-dependent delays, J. Dynam. Differential Equations 23 (2011), 843-884.

[14] F. Hartung, T. Krisztin, H.O. Walther, J. Wu, Functional Differential Equations with StateDependent Delays: Theory and Applications, Handbook of Differential Equations: Ordinary Differential Equations vol. 3, Elsevier, North-Holland, 2006, 435-545.

[15] X. He, R. de la Llave, Construction of quasi-periodic solutions of state-dependent delay differential equations by the parameterization method I: finitely differentiable, hyperbolic case,e J. Dynam. Differential Equations, online since February 2016.

[16] X. He, R. de la Llave, Construction of quasi-periodic solutions of state-dependent delay differential equations by the parameterization method II: analytic case J. Differential Equations 261 (3) (2016), 2068-2108.

[17] R. Johnson, J. Moser, The rotation number for almost periodic potentials, Comm. Math. Phys. 84 (1982), 403-438.

[18] R. Johnson, R. Obaya, S. Novo, C. Núñez, R. Fabbri, Nonautonomous Linear Hamiltonian Systems: Oscillation, Spectral Theory and Control, Developments in Mathematics 36, Springer, 2016.

[19] Q. Hu, J. Wu, X. Zou, Estimates of periods and global continua of periodic solutions for state-dependent delay equations, SIAM J. Math. Anal. 44 (4) (2012), 2401-2427.

[20] Q. Hu, J. Wu, Global Hopf bifurcation for differential equations with state-dependent delay, J. Differential Equations 248 (2010), 2801-2840.

[21] P. Kloeden, M. Rassmussen, Nonautonomous Dynamical Systems, Mathematical Surveys and Monographs, Amer. Math. Soc., 2011.

[22] T. Krisztin, A. Rezounenko, Parabolic partial differential equations with discrete statedependent delay: Classical solutions and solution manifold J. Differential Equations 260 (2016), 4454-4472.

[23] J. Mallet-Paret, R.D. Nussbaum, Stability of periodic solutions of state-dependent delaydifferential equations, J. Differential Equations 250 (2011), 4085-4103.

[24] I. Maroto, C. Núñez, R. Obaya, Dynamical properties of nonautonomous functional differential equations with state-dependent delay, Discrete Contin. Dynam. Systems, Ser. A 37 (7) (2017), 3939-3961.

[25] I. Maroto, C. Núñez, R. Obaya, Exponential stability for nonautonomous functional differential equations with state-dependent delay, Discrete Contin. Dynam. Systems, Ser. B 22 (8) (2017), 3167-3197.

[26] K.R. Meyer, G.R. Sell, Melnikov transforms, Bernoulli bundles, and almost periodic perturbations, Trans. Amer. Math. Soc. 314 (1) (1989), 63-105. 
[27] S. Novo, R. Obaya, A.M. Sanz, Exponential stability in non-autonomous delayed equations with applications to neural networks, Discrete Contin. Dyn. Syst., Ser. A 18 (2007), 517536 .

[28] S. Novo, R. Obaya, A.M. Sanz, Stability and extensibility results for abstract skew-product semiflows, J. Differential Equations 235 (2007), 623-646.

[29] R.J. Sacker, G.R. Sell, Lifting Properties in Skew-Products Flows with Applications to Differential Equations, Mem. Amer. Math. Soc. 190, Amer. Math. Soc., Providence, 1977.

[30] R.J. Sacker, G.R. Sell, Dichotomies for linear evolutionary equations in Banach spaces, J. Differential Equations 113 (1994), 17-67.

[31] G.R. Sell, Topological Dynamics and Differential Equations, Van Nostrand Reinhold, London, 1971.

[32] W. Shen, Y. Yi, Almost Automorphic and Almost Periodic Dynamics in Skew-Product Semiflows, Mem. Amer. Math. Soc. 647, Amer. Math. Soc., Providence, 1998.

[33] H.L. Smith, Monotone Dynamical Systems. An introduction to the Theory of Competitive and Cooperative Systems, Amer. Math. Soc., Providence, 1995.

[34] P. van den Driessche, X Zou, Global attractivity in delayed Hopfield neural network models, SIAM J. Appl. Math. 58 (6) (1998), 1878-1890.

[35] H-O. Walther, Differential equation with locally bounded delay, J. Differential Equations 252 (4) (2012), 3001-3039.

[36] H-O. Walther, Evolution systems for differential equations with variable time lags, J. Math. Sci. 202 (6) (2014), 911-933.

[37] E. Winston, Uniqueness of the zero solution for differential equations with statedependence, J. Differential Equations 7 (1970), 395-405.

[38] J. Wu, Introduction to Neural Dynamics and Signal Transmission Delay, Nonlinear Analysis and Aplications 6, Walter de Gruyter, Berlin, New York, 2001.

(I. Maroto, C. Núñez, R. Obaya) Departamento de Matemática Aplicada, Universidad de Valladolid, Paseo del Cauce 59, 47011 Valladolid, Spain

(C. Elia) Dipartimento di Matematica, Università degli Studi di Bari, Via Orabona 4, 70125 BARI, ITALY

E-mail address, Cinzia Elia: cinzia.elia@uniba.it

E-mail address, Ismael Maroto: ismmar@eii.uva.es

E-mail address, Carmen Núñez: carnun@wmatem.eis.uva.es

E-mail address, Rafael Obaya: rafoba@wmatem.eis.uva.es 